OPEN ACCESS

Edited by:

Hannetz Roschzttardtz,

Pontificia Universidad Católica

de Chile, Chile

Reviewed by:

Ping Lan,

Institute of Soil Science (CAS), China

Louis Grillet,

Academia Sinica, Taiwan

*Correspondence:

Francisco J. Romera ag1roruf@uco.es

Specialty section:

This article was submitted to

Plant Nutrition,

a section of the journal

Frontiers in Plant Science

Received: 19 March 2018

Accepted: 23 August 2018

Published: 11 September 2018

Citation:

García MJ, Corpas FJ, Lucena C, Alcántara E, Pérez-Vicente R, Zamarreño ÁM, Bacaicoa $E$, García-Mina JM, Bauer P and Romera FJ (2018) A Shoot Fe

Signaling Pathway Requiring the OPT3 Transporter Controls GSNO Reductase and Ethylene in Arabidopsis thaliana Roots.

Front. Plant Sci. 9:1325.

doi: 10.3389/fpls.2018.01325

\section{A Shoot Fe Signaling Pathway Requiring the OPT3 Transporter Controls GSNO Reductase and Ethylene in Arabidopsis thaliana Roots}

María J. García', Francisco J. Corpas², Carlos Lucena ${ }^{3}$, Esteban Alcántara3, Rafael Pérez-Vicente', Ángel M. Zamarreño ${ }^{4}$, Eva Bacaicoa ${ }^{4}$, José M. García-Mina ${ }^{4}$, Petra Bauer ${ }^{5}$ and Francisco J. Romera ${ }^{3 *}$

${ }^{1}$ Department of Botany, Ecology and Plant Physiology, Campus de Excelencia Internacional Agroalimentario, Universidad de Córdoba, Córdoba, Spain, ${ }^{2}$ Department of Biochemistry, Cell and Molecular Biology of Plants, Estación Experimental del Zaidin, Spanish National Research Council, Granada, Spain, ${ }^{3}$ Department of Agronomy, Campus de Excelencia Internacional Agroalimentario, Universidad de Córdoba, Córdoba, Spain, ${ }^{4}$ Department of Environmental Biology, Faculty of Sciences, University of Navarra, Pamplona, Spain, ${ }^{5}$ Institute of Botany, University of Düsseldorf, Düsseldorf, Germany

Ethylene, nitric oxide (NO) and glutathione (GSH) increase in Fe-deficient roots of Strategy I species where they participate in the up-regulation of $\mathrm{Fe}$ acquisition genes. However, S-nitrosoglutathione (GSNO), derived from NO and GSH, decreases in Fe-deficient roots. GSNO content is regulated by the GSNO-degrading enzyme $S$-nitrosoglutathione reductase (GSNOR). On the other hand, there are several results showing that the regulation of Fe acquisition genes does not solely depend on hormones and signaling molecules (such as ethylene or NO), which would act as activators, but also on the internal Fe content of plants, which would act as a repressor. Moreover, different results suggest that total $\mathrm{Fe}$ in roots is not the repressor of $\mathrm{Fe}$ acquisition genes, but rather the repressor is a Fe signal that moves from shoots to roots through the phloem [hereafter named LOng Distance Iron Signal (LODIS)]. To look further in the possible interactions between LODIS, ethylene and GSNOR, we compared Arabidopsis WT Columbia and LODIS-deficient mutant opt3-2 plants subjected to different Fe treatments that alter LODIS content. The opt3-2 mutant is impaired in the loading of shoot Fe into the phloem and presents constitutive expression of Fe acquisition genes. In roots of both Columbia and opt3-2 plants we determined 1-aminocyclopropane1-carboxylic acid (ACC, ethylene precursor), expression of ethylene synthesis and signaling genes, and GSNOR expression and activity. The results obtained showed that both 'ethylene' (ACC and the expression of ethylene synthesis and signaling genes) and 'GSNOR' (expression and activity) increased in Fe-deficient WT Columbia roots. Additionally, Fe-sufficient opt3-2 roots had higher 'ethylene' and 'GSNOR' than Fesufficient WT Columbia roots. The increase of both 'ethylene' and 'GSNOR' was not related to the total root Fe content but to the absence of a Fe shoot signal (LODIS), and was associated with the up-regulation of Fe acquisition genes. The possible relationship between GSNOR(GSNO) and ethylene is discussed.

Keywords: ethylene, glutathione (GSH), iron, long distance iron signal (LODIS), nitric oxide (NO), phloem, $S$-nitrosoglutathione (GSNO), S-nitrosoglutathione reductase (GSNOR) 


\section{INTRODUCTION}

Iron $(\mathrm{Fe})$ is abundant in most soils, mainly as $\mathrm{Fe}^{3+}$, although its availability to plants is low, especially in calcareous soils (Römheld and Marschner, 1986). Based on the mechanisms used by plant roots to facilitate mobilization and uptake of $\mathrm{Fe}$, plants are classified into Strategy I species and Strategy II species (Römheld and Marschner, 1986; Ivanov et al., 2012; Kobayashi and Nishizawa, 2012). Dicots, such as Arabidopsis and pea (Pisum sativum), are Strategy I species and reduce $\mathrm{Fe}^{3+}$ to $\mathrm{Fe}^{2+}$, by means of a ferric reductase (encoded by FRO2 in Arabidopsis) at the root surface, prior to its subsequent uptake through a $\mathrm{Fe}^{2+}$ transporter (encoded by IRT1 in Arabidopsis; Ivanov et al., 2012; Kobayashi and Nishizawa, 2012). When grown under Fe deficiency, Strategy I species develop several physiological and morphological responses, mainly in roots, which favor $\mathrm{Fe}$ acquisition and are generally known as Fe deficiency responses. Among the physiological responses are the up-regulation of the ferric reductase and the $\mathrm{Fe}^{2+}$ transporter genes, as well as many other Fe-related genes (Ivanov et al., 2012; Kobayashi and Nishizawa, 2012; Brumbarova et al., 2015; Lucena et al., 2015). In the last years, several transcription factors (TFs) that participate in the activation of these genes have been found (Ivanov et al., 2012; Kobayashi and Nishizawa, 2012; Brumbarova et al., 2015; Zhang et al., 2015; Li et al., 2016; Liang et al., 2017). In Arabidopsis, the master regulator of most of the Ferelated genes is FIT (bHLH29), homolog of the tomato FER gene (Bauer et al., 2007 and references therein). The FIT regulatory network comprises other bHLH TFs of the Ib subgroup, such as bHLH38, bHLH39, bHLH100, and bHLH101. All of them have redundant functions and can interact with FIT to form heterodimers that activate the expression of the Fe acquisition genes FRO2 and IRT1 (Yuan et al., 2008; Wang et al., 2013; Brumbarova et al., 2015). FIT is induced in roots in response to Fe deficiency while the other Ib bHLH genes cited above are induced in both roots and leaves in response to Fe deficiency (Brumbarova et al., 2015 and references therein). Lately, it has been found that, under Fe-deficiency conditions, IVc subgroup bHLH TFs, like bHLH105 and bHLH115, activate the expression of FIT/bHLH38/39/100/101 (Zhang et al., 2015; Li et al., 2016; Liang et al., 2017).

The regulation of the above TFs and genes is not totally understood, but several works support a role for hormones and signaling molecules in the activation of FIT (and other Ib bHLH TFs) and, consequently, in the up-regulation of the ferric reductase and the $\mathrm{Fe}^{2+}$ transporter genes. Among them are auxin, ethylene, salicylic acid, nitric oxide (NO), sucrose, and glutathione (GSH). All of them increase in Fe-deficient roots although their precise roles and interactions are not totally known (Zaharieva and Abadía, 2003; Zaharieva et al., 2004; Lucena et al., 2006, 2015; Graziano and Lamattina, 2007; Waters et al., 2007; Bacaicoa et al., 2009, 2011; Chen et al., 2010; García et al., 2010, 2011; Lingam et al., 2011; Meiser et al., 2011; Romera et al., 2011, 2017; Koen et al., 2012; Yang et al., 2014; Shanmugam et al., 2015; Lin et al., 2016; Shen et al., 2016; Li and Lan, 2017; Kailasam et al., 2018). There are also some hormones, such as cytokinins and jasmonates, that have been involved in the suppression of Fe deficiency responses (Séguéla et al., 2008; Maurer et al., 2011). Besides the activation of Fe-related genes, auxin, ethylene, and NO have also been involved in the regulation of morphological responses to Fe deficiency, such as the development of subapical root hairs (Romera et al., 2011, 2017; Lucena et al., 2015; Li and Lan, 2017). Additionally, ethylene is involved in restricting the suberization of the endodermis under Fe deficiency (Barberon et al., 2016), and NO and GSH are involved in improving Fe availability inside plants (Graziano et al., 2002; Ramírez et al., 2013).

In a previous work, García et al. (2011) showed that ethylene can induce $\mathrm{NO}$ accumulation in the subapical region of the roots, where most Fe responses are located. On the other hand, they found that NO can upregulate many ethylene synthesis and signaling genes in roots. This mutual and positive influence between ethylene and NO has also been described in the development of root hairs under Mg deficiency (Liu et al., 2017) and in other physiological processes (García et al., 2011 and references therein; Lin et al., 2013). Both ethylene and NO greatly activate the expression of Fe acquisition genes in plants grown with low levels of $\mathrm{Fe}$ (or without $\mathrm{Fe}$ ), but have much less effect in plants grown with high levels of $\mathrm{Fe}$ (Lucena et al., 2006; Graziano and Lamattina, 2007; Chen et al., 2010; García et al., 2011). Similar results have also been found when other activators of Fe acquisition genes, like auxin (Chen et al., 2010) or sucrose (Lin et al., 2016), have been applied to plants grown with different Fe levels. All these results suggest that the up-regulation of $\mathrm{Fe}$ acquisition genes does not solely depend on hormones and signaling molecules (such as ethylene, auxin, sucrose, or $\mathrm{NO}$ ), that would act as activators, but also on the internal Fe content of plants, that would act as a repressor (Lucena et al., 2006; García et al., 2011, 2013; Romera et al., 2011). However, different results suggest that total $\mathrm{Fe}$ in roots is not the repressor of Fe acquisition genes. As examples, Arabidopsis opt3, frd3, nas $4 x-1$ and bts 3 mutants, Arabidopsis AtHSCB overexpressing lines, tomato $c h l n$ mutant, and pea $d g l$ and $b r z$ mutants, all of them present constitutive activation of $\mathrm{Fe}$ acquisition genes when grown under Fe sufficiency despite the high accumulation of $\mathrm{Fe}$ inside their roots (García et al., 2013 and references therein; Romera et al., 2015; Leaden et al., 2016; Hindt et al., 2017; Khan et al., 2018). Several of the above genotypes are affected, either directly or indirectly, in the transport of Fe through the phloem, which suggests that phloem Fe could be a key factor in the repression of Fe acquisition genes (García et al., 2013; Khan et al., 2018). In any case, there is no doubt that shoots play a very important role in the regulation of $\mathrm{Fe}$ acquisition genes in roots (García et al., 2013 and references therein; Mendoza-Cózatl et al., 2014; Zhai et al., 2014; Gayomba et al., 2015). In accordance with this, Khan et al. (2018) have recently demonstrated that the leaf vasculature respond to Fe deficiency considerably faster than roots, and Kumar et al. (2017) have found, by using reciprocal shoot/root grafts between Arabidopsis ysllysl3 double mutant and WT plants, that the ability to express the Fe deficiency responses depends on the genotype of the shoot.

OPT3 (OligoPeptide Transporter3) is the main transporter implicated in the loading of shoot Fe into the phloem (MendozaCózatl et al., 2014; Zhai et al., 2014; Kumar et al., 2017; 
Khan et al., 2018). In supporting this view, it should be noted that the foliar application of $\mathrm{Fe}$ did not inhibit the expression of $\mathrm{Fe}$ acquisition genes in Fe-deficient Arabidopsis opt3-2 roots while it did in Fe-deficient Arabidopsis WT roots (García et al., 2013). Furthermore, phloem sap measurements in opt3 mutants show a $50 \%$ reduction in Fe content (Khan et al., 2018). OPT3 is expressed in the plasma membrane of phloem cells, mainly in shoots, and its shoot-specific expression is sufficient to complement the Fe-deficiency response in opt3-2 roots (Mendoza-Cózatl et al., 2014; Zhai et al., 2014; Gayomba et al., 2015). These results suggest that OPT3 plays an important role in the iron-signaling network between leaves and roots (García et al., 2013; Mendoza-Cózatl et al., 2014; Zhai et al., 2014; Gayomba et al., 2015). The precise substrate of the OPT3 transporter is not yet clear. Zhai et al. (2014) found that OPT3 can transport $\mathrm{Fe}^{2+}$ ions when expressed in Xenopus oocytes. However, while Wintz et al. (2003) found that OPT3 was able to rescue the fet $3 f e t 4$ strain of yeast, impaired in Fe uptake, Mendoza-Cózatl et al. (2014) found that not. Even if OPT3 transports Fe ions, these ions should be chelated in the phloem sap due to the poor solubility of $\mathrm{Fe}$ at the alkaline $\mathrm{pH}$ of this fluid (Gutiérrez-Carbonell et al., 2015). Although OPT3 does not mediate GSH transport in S. cerevisiae (Zhai et al., 2014), other OPT transporters do (Lubkowitz, 2011 and references therein; Zhang et al., 2016 and references therein). Consequently, some proposed chelating agents of Fe are GSH-derived compounds, like $S$-nitrosoglutathione (GSNO) or nitrosyl-iron complexes (NICs, formed from the interaction of Fe, $\mathrm{NO}$ and thiols); also peptides and proteins (Krüger et al., 2002; Ramírez et al., 2011; Rahmanto et al., 2012; Darbani et al., 2013; García et al., 2013; Buet and Simontacchi, 2015). Based on these findings, we suggest

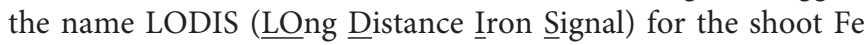
signal related to $\mathrm{OPT} 3$ and moving through the phloem, which causes the repression of Fe acquisition genes in roots. Although the LODIS nature is still unknown, it is possible to investigate its consequences by comparing the LODIS-deficient mutant opt3-2 and the WT cultivar Columbia, and by comparing plants treated or not with foliar application of Fe.

Another important question, which remains unsolved, is how the Fe status is perceived by the roots. At this point, it has been proposed that Fe (probably, LODIS or a LODIS-derived signal) could be sensed by the BRUTUS (BTS) protein in Strategy I plants (Kobayashi and Nishizawa, 2014). BTS is a homolog of HRZs, known Fe sensors in rice (Kobayashi and Nishizawa, 2014). The current data suggests that BTS acts as a negative regulator of Fe responses (Zhang et al., 2015; Hindt et al., 2017). This would explain why Fe-sufficient $b t s-2$ and $b t s-3$ roots present higher expression of $\mathrm{Fe}$ acquisition genes than $\mathrm{Fe}$-sufficient WT roots (Zhang et al., 2015; Hindt et al., 2017).

To integrate both positive and negative signals in the regulation of Fe acquisition genes in roots, Lucena et al. (2006) proposed a model that implicated both LODIS and ethylene in such a regulation. Subsequently, this model has been extended to other positive signals besides ethylene, like NO and auxin (García et al., 2011; Romera et al., 2011, 2017). According to this model, auxin/ethylene/NO would act as activators of the expression of Fe acquisition genes in roots, while LODIS would act to repress their expression (García et al., 2011; Romera et al., 2011, 2017). This model does not exclude the role of other positive and negative signals (most of them reviewed by García et al., 2015; Romera et al., 2017). The way LODIS represses the expression of Fe acquisition genes is not totally known, but several results suggest that it could negatively affect ethylene action. García et al. (2013) found that ACC up-regulated the expression of Fe acquisition genes when applied to roots of Fe-deficient plants but not when applied simultaneously with foliar Fe. This result suggests that LODIS could block ethylene action, but does not preclude an additional negative effect of LODIS on ethylene production.

One of the objectives of this work was to study the role of LODIS on ethylene synthesis in roots. For this, we determined 1aminocyclopropane-1-carboxylic acid (ACC) concentration and the expression of the key ethylene synthesis genes SAM1, ACS6, and ACO2 in roots of Arabidopsis WT Columbia plants subjected to different Fe treatments that alter LODIS content. It should be noted that ACC content and the expression of the above ethylene synthesis genes increase under Fe deficiency (García et al., 2010; Gutiérrez-Carbonell et al., 2015; Lucena et al., 2015 and references therein; Ye et al., 2015; Romera et al., 2017 and references therein). In a parallel study, similar determinations were done in the Arabidopsis LODIS-deficient mutant opt32, and in other Arabidopsis mutants that behave like LODISdeficient, such as frd3-3 and nas $4 x-1$. All of these mutants have constitutive activation of $\mathrm{Fe}$ acquisition genes when grown under Fe sufficient conditions (see section "Materials and Methods"; Rogers and Guerinot, 2002; Klatte et al., 2009; García et al., 2013). A second objective of this work was to study the possible relationship of $S$-nitrosoglutathione reductase (GSNOR) with Fe deficiency and, more specifically, with LODIS. Both GSH and NO increase in roots under Fe deficiency and have been implicated in the activation of $\mathrm{Fe}$ responses (Zaharieva and Abadía, 2003; Zaharieva et al., 2004; Graziano and Lamattina, 2007; Bacaicoa et al., 2009; Chen et al., 2010; García et al., 2011; Koen et al., 2012; Shanmugam et al., 2015; Kailasam et al., 2018). However, $S$-nitrosoglutathione (GSNO), the most abundant lowmolecular-weight $S$-nitrosothiol in plants (Liu et al., 2018), and which is derived from GSH and NO (Corpas et al., 2013), decreases under Fe deficiency in Arabidopsis roots (Shanmugam et al., 2015; Kailasam et al., 2018). Since GSNOR regulates GSNO content by decomposing it to oxidized glutathione (GSSG) and $\mathrm{H}_{3} \mathrm{~N}$ (Leterrier et al., 2011; Corpas et al., 2013), we wanted to know whether GSNOR increases in roots under Fe deficiency. For this, we determined GSNOR expression and activity under Fe deficiency and also under different Fe treatments and genetic backgrounds that could influence LODIS levels.

The results show that ethylene (ACC and the expression of ethylene synthesis genes) and GSNOR (expression and activity) increase under conditions that restrict LODIS accumulation in roots, such as Fe deficiency in WT or Fe sufficiency in the LODISdeficient mutant opt3-2, while they decrease under conditions that favor its accumulation, such as Fe sufficiency or foliar application of Fe in WT. The up-regulation of GSNOR under Fe deficiency in the WT suggests that lower GSNO levels in roots could be a prerequisite for the up-regulation of ethylene synthesis and, consequently, for that of Fe acquisition genes. 


\section{MATERIALS AND METHODS}

\section{Plant Materials, Growth Conditions, and Treatments}

To study the role of LODIS on ethylene and GSNOR in roots, wild-type Arabidopsis [Arabidopsis thaliana (L.) Heynh ecotype Columbia] and pea [Pisum sativum L. cv. Sparkle] plants were used. Additionally, we used some mutants that show constitutive up-regulation of $\mathrm{Fe}$ acquisition genes when grown under $\mathrm{Fe}$ sufficient conditions and that are, or behave like, LODIS-deficient (García et al., 2013 and references therein). These mutants were Arabidopsis opt3-2, frd3-3 and nas $4 x-1$, and pea $d g l$ (Sparkle $[d g l, d g l])$. OPT3 is expressed mainly in shoots, where it could act as a transporter involved in the loading of $\mathrm{Fe}^{2+}$ ions into the phloem (Stacey et al., 2008; Zhai et al., 2014; see Figure 12). The Arabidopsis frd3-3 mutant is impaired in xylem Fe transport (Rogers and Guerinot, 2002; Roschzttardtz et al., 2011; Gayomba et al., 2015) but, as a consequence, it is also defective in the transport of $\mathrm{Fe}$ from shoots to roots through the phloem (it behaves like LODIS-deficient), since less Fe gets into leaves to enter the phloem (Lucena et al., 2006; see Figure 12). The Arabidopsis nas $4 x-1$ mutant is defective in the synthesis of NA, which is involved in Fe loading and unloading of the phloem (Klatte et al., 2009; Schuler et al., 2012). Both frd3-3 and nas $4 x-1$ mutants show chlorosis when grown under Fe-sufficient conditions (Lucena et al., 2006; Klatte et al., 2009). In relation to the pea $d g l$ mutant, the specific gene related to this mutation has not been identified yet. However, several results suggest that the $d g l$ mutant phenotype may be related to defects in phloem Fe transport since it behaves like a LODIS-deficient mutant (García et al., 2013; Romera et al., 2015). Although the $d g l$ mutant originated from the DGV (Dippes Gelbe Viktoria) cultivar, we used a near isogenic line homozygous for the $d g l$ mutation that was introgressed into the Sparkle cultivar and described here as Sparkle [dgl, $d g l]$ (Marentes and Grusak, 1998).

Arabidopsis and pea plants were grown on aerated nutrient solution in a growth chamber, as previously described (Lucena et al., 2006, 2007). When appropriate, plants were transferred to the different treatments. The treatments imposed were: $+\mathrm{Fe}$ : nutrient solution with $40 \mu \mathrm{M}$ Fe-EDDHA, except for pea WT Sparkle, that was $20 \mu \mathrm{M}$ Fe-EDDHA, and for pea $d g l$, that was $3 \mu \mathrm{M}$ Fe-EDDHA (this mutant grows adequately with $2-3 \mu \mathrm{M}$ Fe-EDDHA concentration but presents symptoms of Fe toxicity when grown with higher levels of Fe; Romera et al., 2015); -Fe: nutrient solution without Fe during different times (from 6 to $72 \mathrm{~h}$, depending on experiments); $-\mathrm{Fe}+$ foliarFe: $-\mathrm{Fe}$ treatment during 2 or 3 days and $\mathrm{FeSO}_{4}$ application to leaves during the last $24 \mathrm{~h} ;-\mathbf{P}$ : nutrient solution without $\mathrm{P}$ during 2 days; $-\mathbf{S}$ : nutrient solution without $S$ during 2 days. It should be noted that, in our experimental conditions, the expression of Fe acquisition genes and the ferric reductase activity was weak after $24 \mathrm{~h}$ of $\mathrm{Fe}$ deficiency and reached their maximum after $48 \mathrm{~h}$ of Fe deficiency. $\mathrm{FeSO}_{4}$ was dissolved in deionized water $(1.8 \mathrm{mM})$ and Tween 20 was added as surfactant. Leaves were sprayed once until total moistening. After treatments, root ferric reductase activity was determined as described previously (Lucena et al., 2006). Finally, the roots were collected and kept at $-80^{\circ} \mathrm{C}$ for subsequent analysis of ACC and mRNA levels. In some experiments, GSNOR activity, GSNO and GSH were determined in fresh roots.

\section{ACC Determination}

The extraction, purification and quantification of ACC was carried out using the method described by Mora et al. (2012). Briefly, ACC of roots was extracted with $20 \mu \mathrm{l}$ of $\mathrm{d}_{4}$ ACC [3 $\mu \mathrm{g} / \mathrm{mL}$ in acetonitrile/acetic acid $0.2 \%(90 / 10)]$ and $3 \mathrm{ml}$ of $\mathrm{MeOH} / \mathrm{H}_{2} \mathrm{O} / \mathrm{HCOOH}(15 / 4 / 1, \mathrm{v} / \mathrm{v} / \mathrm{v})$ at $-20^{\circ} \mathrm{C}$. Purification was carried out using a Strata C18-E cartridge (Ref 8B-S001-FBJ, Phenomenex, Torrance, CA, United States) preconditioned with $4 \mathrm{ml}$ of methanol and $2 \mathrm{ml}$ of $\mathrm{MeOH} / \mathrm{H}_{2} \mathrm{O} / \mathrm{HCOOH}(15 / 4 / 1$, $\mathrm{v} / \mathrm{v} / \mathrm{v})$. Finally, the eluted fraction was centrifuged $(10,000 \mathrm{rpm}$, $8 \mathrm{~min}$ ) and injected in the LC/MS/MS systems, and ACC was quantified by HPLC linked to a 3200 QTRAP LC/MS/MS system (Applied Biosystems/MDS Sciex, Ontario, Canada), equipped with a turbo ion spray interface.

\section{GSNOR Activity Determination}

GSNOR activity was assayed spectrophotometrically at $25^{\circ} \mathrm{C}$ by monitoring the oxidation of $\mathrm{NADH}$ at $340 \mathrm{~nm}$ as described by Sakamoto et al. (2002). The root extracts were incubated in an assay mixture containing $20 \mathrm{mM}$ TRIS- $\mathrm{HCl}$ $(\mathrm{pH}$ 8.0), $0.2 \mathrm{mM} \mathrm{NADH}$, and $0.5 \mathrm{mM}$ EDTA, and the reaction was started by adding GSNO (Calbiochem) to the mixture at a final concentration of $400 \mu \mathrm{M}$. The activity was expressed as nmol NADH consumed $\mathrm{min}^{-1} \mathrm{mg}^{-1}$ protein $\left(\varepsilon_{340}=6.22 \mathrm{mM}^{-1} \mathrm{~cm}^{-1}\right)$.

\section{Quantification of GSNO and GSH by Liquid Chromatography Electrospray Ionization Mass Spectrometry (LC-ES/MS)}

Arabidopsis samples $(300 \mathrm{mg}$ ) were ground using a mortar and pestle in the presence of $1 \mathrm{ml}$ of $0.1 \mathrm{M} \mathrm{HCl}$. Homogenates were centrifuged at $15,000 \mathrm{~g}$ for $20 \mathrm{~min}$ at $4^{\circ} \mathrm{C}$. The supernatants were collected and filtered through $0.22-\mathrm{mm}$ polyvinylidene fluoride filters and immediately analyzed. All procedures were carried out at $4^{\circ} \mathrm{C}$ and were protected from light to avoid potential degradation of the analytes (GSNO and GSH). The LC-ES/MS system consisted of a Waters Allience 2695 HPLC system connected to a Micromass Quattro micro API triple quadrupole mass spectrometer, both obtained from the Waters Corporation. HPLC was carried out using an Atlantis ${ }^{\otimes}$ T3 $3 \mu \mathrm{m} 2.1 \mathrm{~mm} \times 100 \mathrm{~mm}$ Column obtained from the Waters Corporation. The Micromass Quattro Micro API mass spectrometer was used in positive electrospray ionization mode for detection and quantification of GSNO and GSH (Airaki et al., 2011; Leterrier et al., 2012).

\section{qRT-PCR Analysis}

Roots were ground to a fine powder with a mortar and pestle in liquid nitrogen. Total RNA was extracted using the Tri Reagent solution (Molecular Research Center, Inc., Cincinnati, OH, United States) according to the manufacturer's 
instructions. M-MLV reverse transcriptase (Promega, Madison, WI, United States) was used to generate cDNA from $3 \mu \mathrm{g}$ of DNase-treated root RNA as the template and random hexamers as the primers.

The study of gene expression by qRT-PCR was performed by using a qRT-PCR Bio-Rad CFX connect thermal cycler and the following amplification profile: initial denaturation and polymerase activation $\left(95^{\circ} \mathrm{C}\right.$ for $\left.3 \mathrm{~min}\right)$, amplification and quantification repeated 40 times $\left(90^{\circ} \mathrm{C}\right.$ for $10 \mathrm{~s}, 57^{\circ} \mathrm{C}$ for $15 \mathrm{~s}$ and $72^{\circ} \mathrm{C}$ for $30 \mathrm{~s}$ ), and a final melting curve stage of 65 to $95^{\circ} \mathrm{C}$ with increment of $0.5^{\circ} \mathrm{C}$ for $5 \mathrm{~s}$, to ensure the absence of primer dimer or non-specific amplification products. PCR reactions were set up in $20 \mu \mathrm{l}$ of SYBR Green Bio-RAD PCR Master Mix, following the manufacturer's instructions. Controls containing water instead of cDNA were included to check for contamination in the reaction components. Gene-specific primers (Table 1) were designed by using the Primer-BLAST software from the NCBI site. Standard dilution curves were performed for each primer pair to confirm appropriate efficiency of amplification $(\mathrm{E}=100 \pm 10 \%)$. Constitutively expressed SAND1 and YLS8 genes, which do not respond to changes in the Fe conditions (Han et al., 2013), were used as reference genes to normalize qRT-PCR results. The relative expression levels were calculated from the threshold cycles $(\mathrm{Ct})$ values and the primer efficiencies by the Pfaffl method (Pfaffl, 2001). Each PCR analysis was conducted on three biological replicates and each PCR reaction repeated twice.

\section{Statistical Analysis}

All experiments were repeated at least twice and representative results are presented. The values of qRT-PCR represent the mean \pm SE of three independent biological replicates. The values of other determinations (ACC, GSNO, GSH, ferric reductase

TABLE 1 | Primer pairs for Arabidopsis genes.

\begin{tabular}{ll}
\hline Gene & Sequence 5'-3' $^{\prime}$ \\
\hline AtFRO2 & Forward: TGGTTGCCACATCTGCGTAT \\
(At1g01580) & Reverse: TCGATATGGTGTGCGACTT \\
AtIRT1 & Forward: TGTCTCTITGCAATCTGTCCA \\
(At4g19690) & Reverse: AGGAGCTCCAACACCAATCA \\
AtFIT & Forward: CCCTGTTCATAGACGAGAACC \\
(At2g28160) & Reverse: TTCATCTTCTTCACCACCGGC \\
AtbHLH38 & Forward: AAAATGTGTGCATTAGTCCCTT \\
(At3g56970) & Reverse: AGTCTGTGGTACCGTCAATTCAA \\
AtGSNOR1 & Forward: GGTCTCTTCCTTGTATCTAG \\
(At3g56980) & Reverse: GCATTCACGACACTCAGCTTG \\
AtSAM1 & Forward: TAATCTCCCATCGAAGCAGCAG \\
(At1g02500) & Reverse: CAACTTGCTGTCAGGGTCTTG \\
AtACO2 & Forward: AGCAACCCTCTCTCATTCTACA \\
(At1g62380) & Reverse: AGCTTGGACAAGTCTACTACTGG \\
AtACS6 & Forward: ATTGTCTAAAATCGCCTCCGGT \\
(At4g11280) & Reverse: CCACAAAGCTGATITCAGCGA \\
AtEIN2 & Forward: ATTCGCACTGATGGGTCTTCTT \\
(At5g03280) & Reverse: CCAAAGATGGCGAACAAATGGT \\
AtEIN3 & Forward: GTCCAGAGCAACCAAACCTCTA \\
(At3g20770) & Reverse: TGTTCCTGGGAACTGGAGATG \\
AtEIL1 & Forward: CCATCTCTGAAGTGTGGGGAT \\
(At2g27050) & Reverse: ACCACAATCAAGAACAGAGCCT \\
&
\end{tabular}
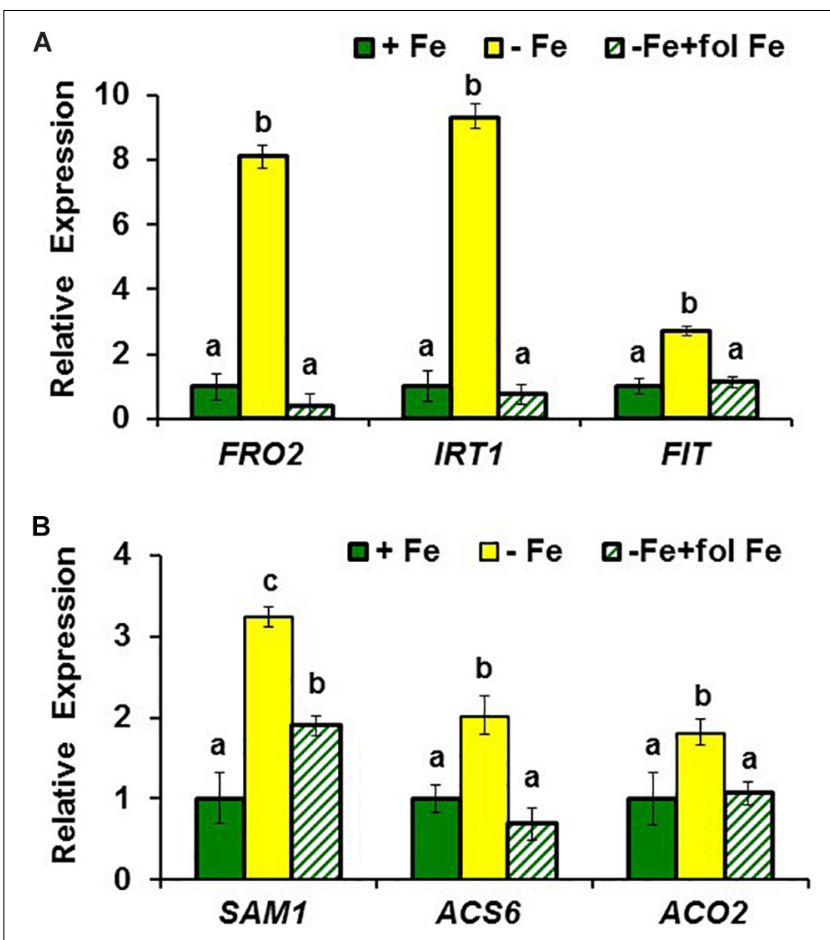

FIGURE 1 | Effect of Fe deficiency and foliar application of Fe on the expression of (A) Fe acquisition genes FRO2, IRT1, and FIT; and (B) ethylene synthesis genes SAM1, ACS6 and ACO2, in roots of Arabidopsis WT Columbia plants. Plants were grown in nutrient solution with $\mathrm{Fe}(+\mathrm{Fe})$. Some of them were transferred to nutrient solution without $\mathrm{Fe} 3$ days before harvest $(-\mathrm{Fe})$. Half of the -Fe plants were sprayed with Fe $24 \mathrm{~h}$ before harvest (-Fe+fol $\mathrm{Fe}$ ). Relative expression was calculated in relation to $+\mathrm{Fe}$. Data represent the mean \pm SE of three independent biological replicates. Within each gene, bars with different letters indicate significant differences $(P<0.05)$. 


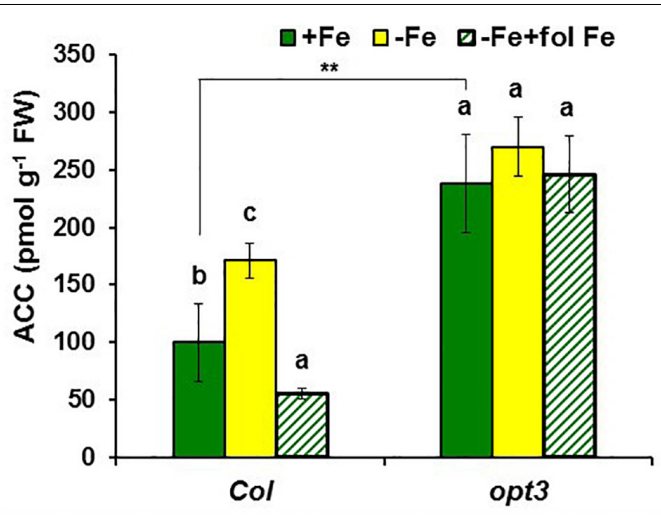

FIGURE 2 | Effect of Fe deficiency and foliar application of Fe on ACC content in roots of Arabidopsis WT Columbia and opt3-2 mutant plants. Treatments as in Figure 1. Data represent the mean \pm SE of six replicates. Within each genotype, bars with different letters indicate significant differences $(P<0.05)$. Significant difference between the $+F e$ treatment from opt3-2 and $\mathrm{Col}$ is also indicated: ${ }^{* *} P<0.05$

expression of all the ethylene synthesis genes studied and ACC production greatly increased (sometimes, more than 2 -fold) in Fe-deficient Columbia roots, in parallel to the expression of the key $\mathrm{Fe}$ acquisition genes FRO2, IRT1, and FIT (Figures 1, 2). On the other hand, the foliar application of $\mathrm{Fe}$ to $\mathrm{Fe}$ deficient Columbia plants greatly repressed the expression of Fe acquisition (Figure 1A) and ethylene synthesis genes (Figure 1B), and ACC production (Figure 2), which suggests that LODIS inhibits ethylene synthesis. This inhibitory effect of LODIS was further supported by the fact that the foliar application of Fe did not significantly inhibit ACC production in the Arabidopsis LODIS-deficient mutant opt3-2 (Figure 2; García et al., 2013; Zhai et al., 2014). This mutant also showed higher ACC concentration in Fe-sufficient roots compared to the WT Columbia (Figure 2), which suggests that total Fe in roots does not control ethylene synthesis. Similar higher ACC levels were found in Fe-sufficient roots of other Arabidopsis mutants that behave like LODIS-deficient ( $f r d 3-3$ and nas $4 x-1$ ) and that present constitutive activation of $\mathrm{Fe}$ acquisition genes under $\mathrm{Fe}$ sufficiency (Figure 3A; García et al., 2013; see section "Materials and Methods").

It should be noted that, although the frd3-3 and opt3-2 mutants usually accumulate more metals than the WT cultivar (Rogers and Guerinot, 2002; Stacey et al., 2008), neither of them showed any symptom of metal toxicity in our experimental conditions (Figure 3B). The frd3-3 and nas $4 x-1$ mutants showed chlorosis, as expected (Figure 3B; see section "Materials and Methods").

The higher ACC content in Fe-sufficient opt3-2 roots than in Columbia roots (Figure 2) was correlated with higher expression of the ethylene synthesis genes SAM1, ACS6 and ACO2, and of the ethylene signaling genes EIN2, EIN3 and EIL1, in the mutant (from 5- to 30-fold; Figure 4). Collectively, these results suggest that ethylene (synthesis and signaling) drastically increases under low LODIS accumulation in roots (i.e., Fe deficiency in WT or Fe sufficiency in the LODIS-deficient mutant opt3-2).

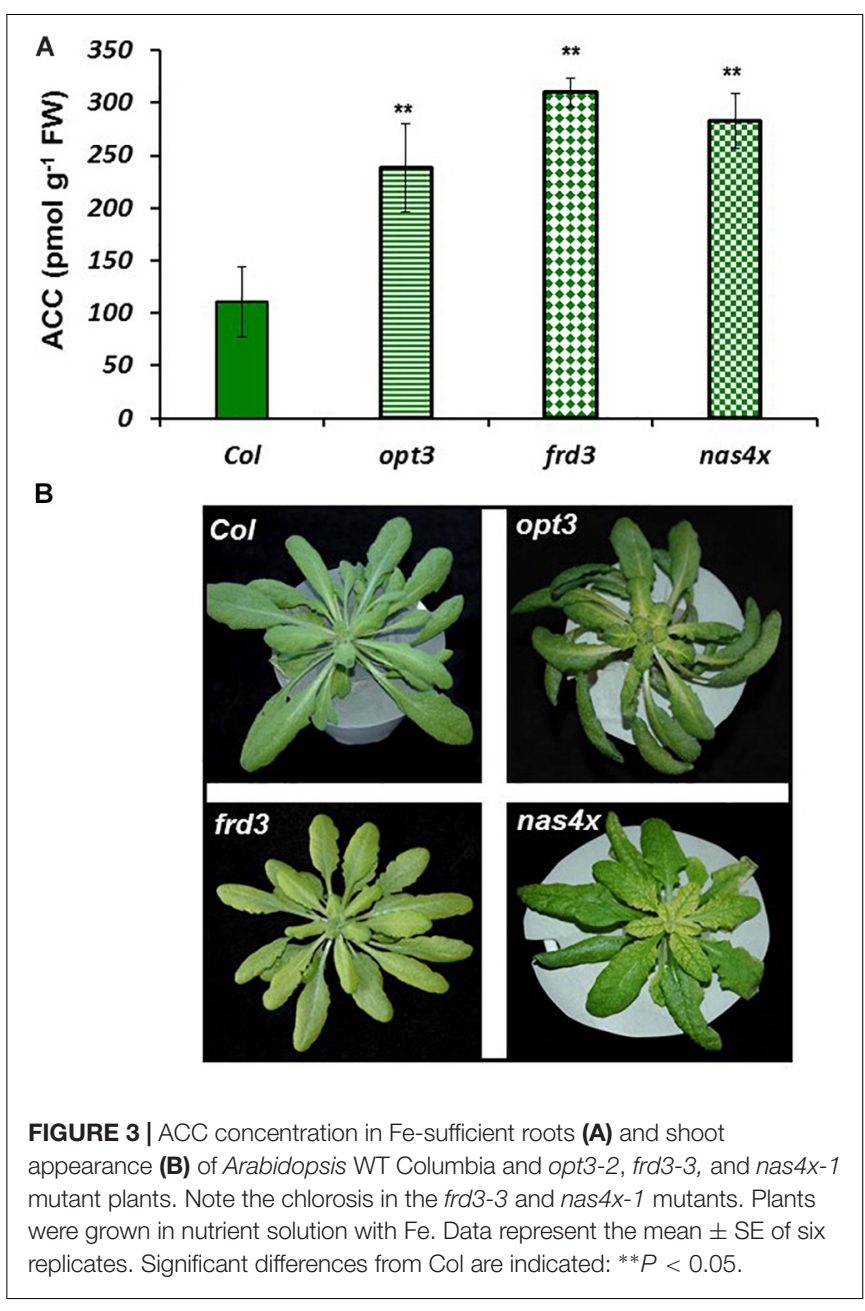

\section{Effect of LODIS on GSNOR Expression and Activity in Roots}

To study the possible relationship of GSNOR with Fe deficiency, we analyzed GSNOR expression and activity under different Fe treatments and genetic backgrounds that could influence LODIS levels. The results showed that GSNOR1 expression increased (more than 10-fold) under Fe deficiency in roots of the WT cultivar Columbia (Figure 5A). This up-regulation of GSNOR1 occurred very quickly, after few hours of Fe depletion, and slightly before the up-regulation of the main regulator of the $\mathrm{Fe}$ deficiency responses, FIT (Figure 5B), other Fe acquisition genes (bHLH38, IRT1, FRO2; Figures 5C-E), and the enhancement of ferric reductase activity (Figure 5F). In agreement with the GSNOR1 up-regulation, the GSNOR activity significantly increased (approximately 50\%) in Fe-deficient Columbia roots (Figure 6). This activity was restored to normal level upon foliar application of $\mathrm{Fe}$ (Figure 6), which suggests that it could be inhibited by the accumulation of LODIS in roots. To further confirm this possibility, we compared GSNOR1 expression in Fe-sufficient WT and opt3-2 roots. As shown in Figure 7, GSNOR1 expression was much higher (more than 50-fold) in Fe-sufficient opt3-2 roots (LODIS-deficient) 


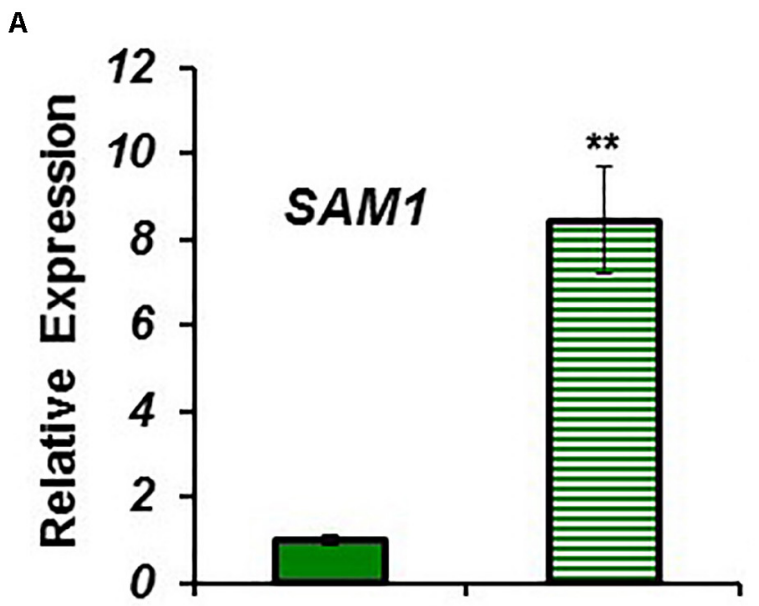

B

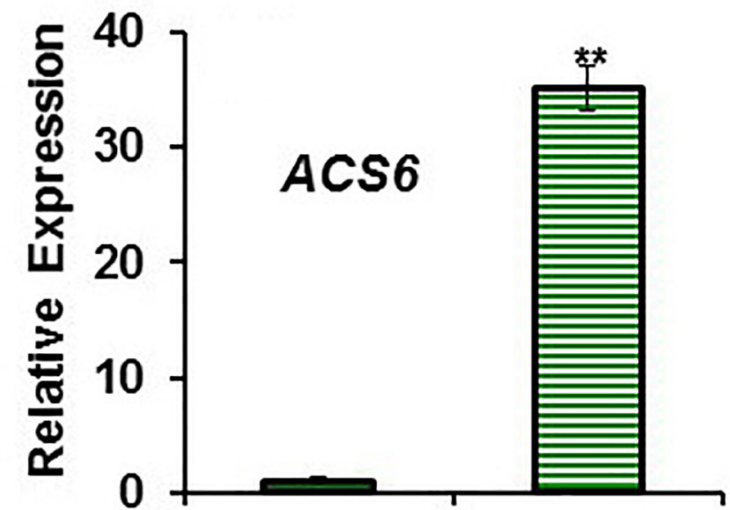

C

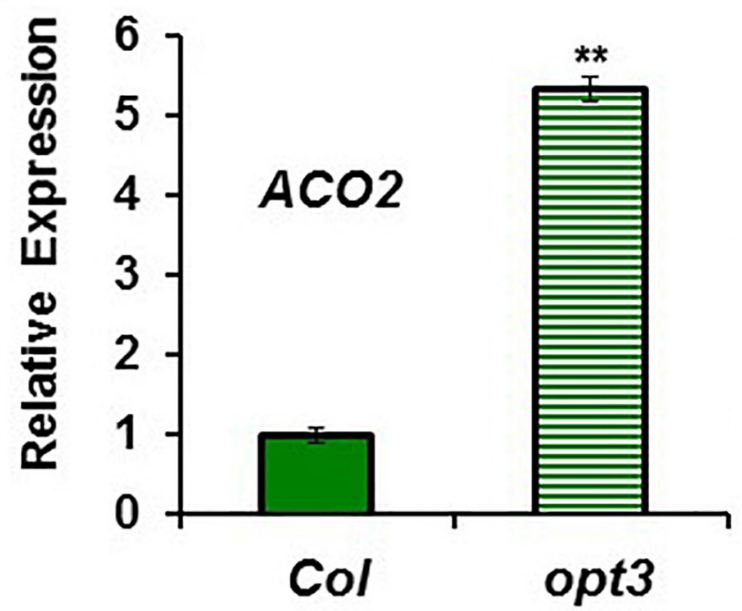

D

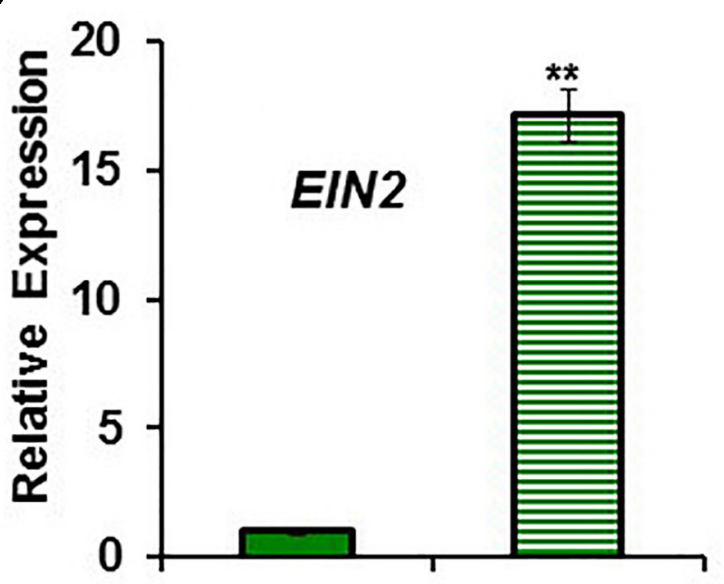

E

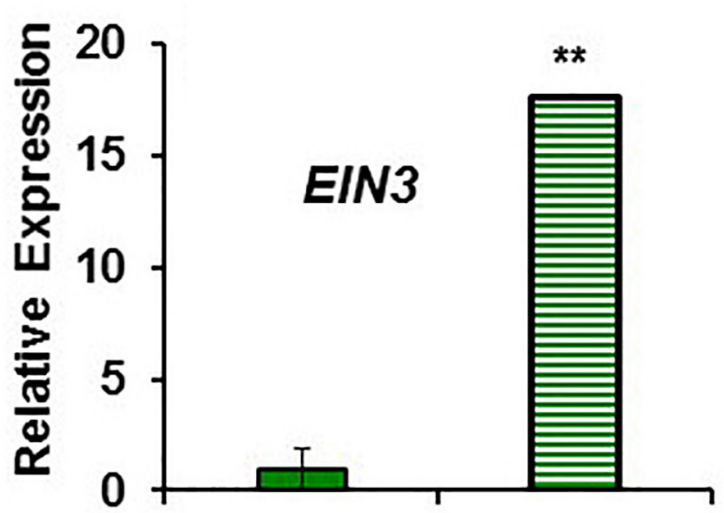

F

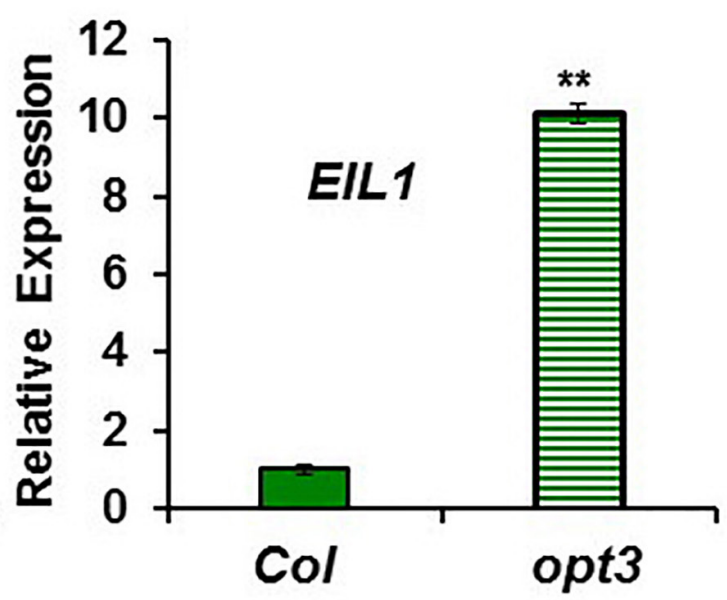

FIGURE 4 | Expression of ethylene synthesis genes (A) SAM1, (B) ACS6 and (C) ACO2, and ethylene signaling genes (D) EIN2, (E) EIN3 and (F) EIL1, in Fe-sufficient roots of Arabidopsis WT Columbia and opt3-2 mutant plants. Plants were grown in nutrient solution with Fe. Relative expression was calculated in relation to Col. Data represent the mean \pm SE of three independent biological replicates. Significant differences between opt3-2 and Col are indicated: ${ }^{* *} P<0.05$.

than in Fe-sufficient Columbia roots, which suggests that its expression depends on LODIS and not on total root $\mathrm{Fe}$ content. The up-regulation of GSNOR1 expression also occurred under other nutrient deficiencies, such as $\mathrm{P}$ deficiency and $\mathrm{S}$ deficiency (Figure 8). Furthermore, GSNOR1 expression was up-regulated by ACC treatment in Fe-sufficient Columbia roots (Figure 9).

In addition to GSNOR, we also determined GSNO concentration in roots of Arabidopsis WT Columbia and opt3-2 plants subjected to different Fe treatments. The results 


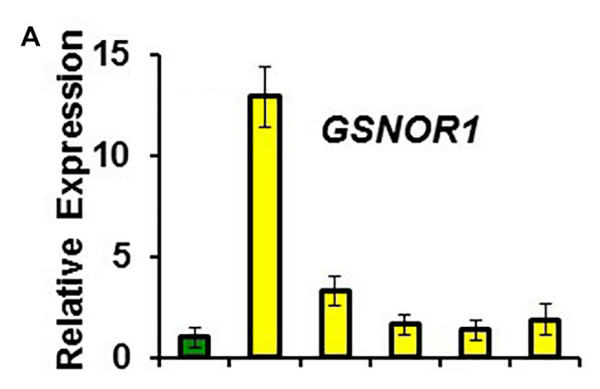

D

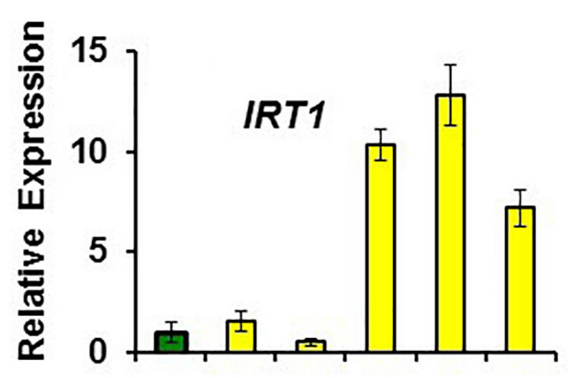

E

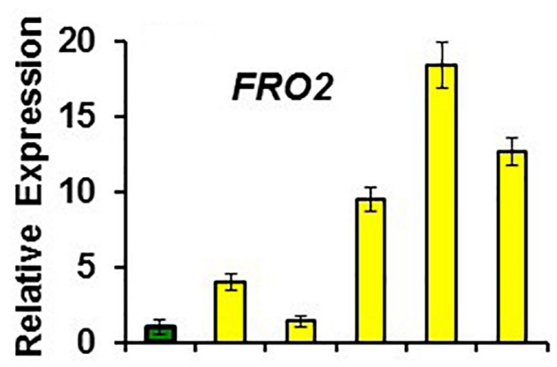

$\mathrm{F}$

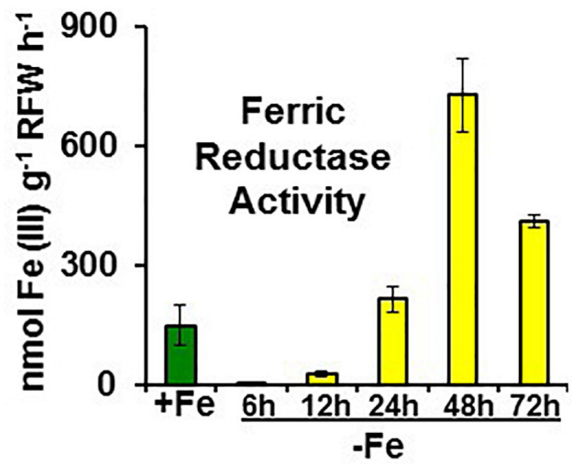

FIGURE 5 | Time course experiment of the effect of Fe deficiency on the expression of (A) GSNOR1, Fe acquisition genes (B) FIT, (C) bHLH38, (D) IRT1 and (E) FRO2), and on (F) ferric reductase activity, in roots of Arabidopsis WT Columbia plants. Plants were grown in nutrient solution with $\mathrm{Fe}$ (+Fe). Some of them were transferred to nutrient solution without Fe $(-\mathrm{Fe}) 6,12,24,48$, or $72 \mathrm{~h}$ before determination of ferric reductase activity or harvest. For genes, data represent the mean \pm SE of three independent biological replicates. Relative expression was calculated in relation to +Fe. For ferric reductase activity, data represent the mean \pm SE of six replicates.

showed a decrease of approximately $30 \%$ of GSNO content in Fe-deficient WT roots and, by contrast, a great increase upon foliar application of Fe (Figure 10). GSNO also decreased in Fe-deficient opt3-2 roots but was less affected by the foliar application of Fe (Figure 10). The lower GSNO content in Fe-deficient roots could be explained by the higher GSNOR expression and activity in these roots (Figures 5, 6). In supporting this view, Fe-sufficient opt3-2 roots, which had higher GSNOR expression than Fe-sufficient WT roots (Figure 7), had lower GSNO content (approximately 50\%; Figure 10). Similar results were also found with the pea LODIS-deficient mutant $d g l$ and its WT cultivar Sparkle. As shown in Figure 11, GSNO content also decreased in Fe-deficient Sparkle roots (approximately 25\%) and, similarly to opt3-2, GSNO content was lower in Fe-sufficient $d g l$ roots (approximately 40\%) than in Fe-sufficient Sparkle roots.

Collectively, all these results suggest that GSNOR (expression and activity) increases, and consequently GSNO decreases, under low LODIS accumulation in roots (i.e., Fe deficiency in WT or Fe sufficiency in LODIS-deficient mutants).

\section{Effect of Fe Deficiency on GSH Content in Roots and Leaves of WT and opt3-2 Mutant Plants}

In our experimental conditions, GSH greatly increased in $\mathrm{Fe}$ deficient WT roots but hardly in Fe-deficient opt3-2 roots (Table 2). In leaves, the most noticeable result was the higher GSH content in the opt3-2 mutant in relation to Columbia under Fe sufficiency (Table 2).

\section{DISCUSSION}

Ethylene increases in Fe-deficient roots, where it activates the expression of key $\mathrm{Fe}$ acquisition genes (reviewed in Lucena et al., 2015; Li and Lan, 2017; Romera et al., 2017). A signal 


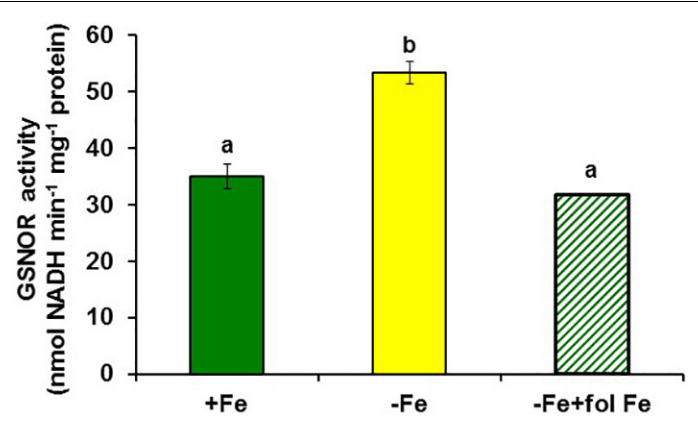

FIGURE 6 | Effect of Fe deficiency and foliar application of Fe on GSNOR activity in roots of Arabidopsis WT Columbia plants. Plants were grown in nutrient solution with $\mathrm{Fe}(+\mathrm{Fe})$ and some of them were transferred to nutrient solution without $\mathrm{Fe} 2$ days before harvest $(-\mathrm{Fe})$. Half of the $-\mathrm{Fe}$ plants were sprayed with $\mathrm{Fe} 24 \mathrm{~h}$ before harvest (-Fe+fol Fe). Data represent the mean \pm SE of six replicates. Bars with different letters indicate significant differences $(P<0.05)$.

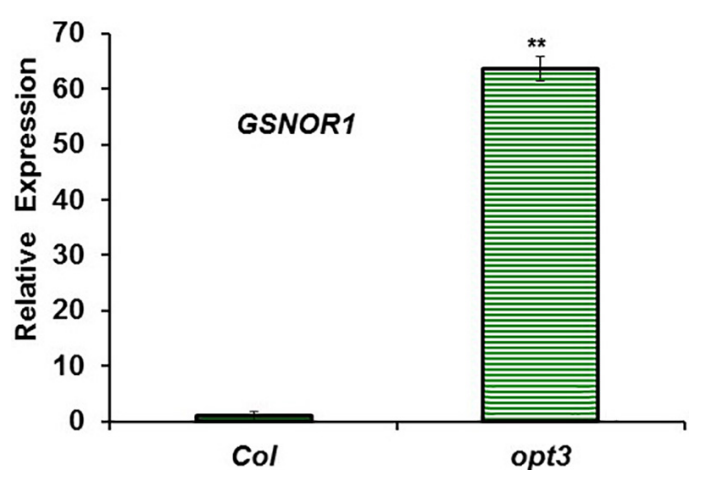

FIGURE 7 | GSNOR1 expression in roots of Fe-sufficient Arabidopsis WT Columbia and opt3-2 mutant plants. Plants were grown in nutrient solution with Fe. Relative expression was calculated in relation to Col. Data represent the mean \pm SE of three independent biological replicates. Significant difference between opt3-2 and Col is indicated: ${ }^{* *} P<0.05$. related to phloem Fe, LODIS, blocks this activating effect (García et al., 2013). However, it is not known whether LODIS could affect ethylene synthesis in roots. Results presented in this work (Figures 1, 2, 3A, 4) support that LODIS does affect ethylene synthesis. The foliar application of Fe greatly decreased the expression of Fe acquisition and ethylene synthesis genes (Figure 1), and ACC production (Figure 2), in Fe-deficient WT Columbia roots. However, in Fe-deficient roots of the LODISdeficient mutant opt3-2 (García et al., 2013; Mendoza-Cózatl et al., 2014; Zhai et al., 2014), foliar application of Fe had almost no effect on ACC content of roots (Figure 2). Moreover, the expression of several ethylene synthesis and signaling genes was much higher in Fe-sufficient opt3-2 roots than in Fe-sufficient Columbia roots (Figure 4). In the same way, Fe-sufficient roots of opt3-2 and other Arabidopsis mutants that behave as LODISdeficient, such as frd3-3 and nas $4 x-1$ (see section "Materials and Methods"), had greater ACC content than Fe-sufficient Columbia roots (Figures 2, 3A). All these results suggest that

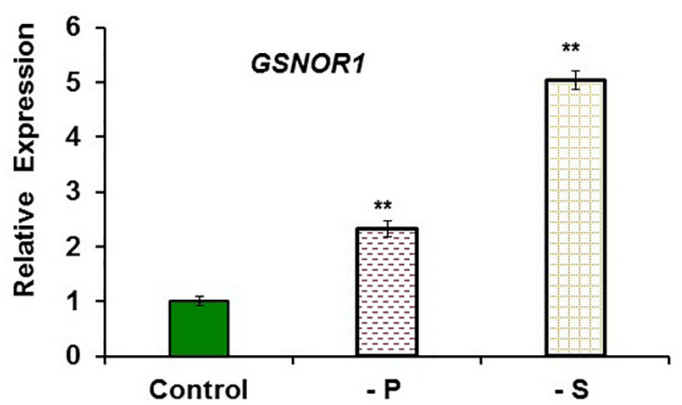

FIGURE 8 | Effect of P or S deficiency on GSNOR1 expression in roots of Arabidopsis WT Columbia plants. Plants were grown in complete nutrient solution (Control). Some of them were transferred to nutrient solution without $P(-P)$ or without $S(-S)$ during the last 2 days. Relative expression was calculated in relation to Control. Data represent the mean $\pm S E$ of three independent biological replicates. Significant differences from the Control treatment are indicated: ${ }^{* *} P<0.05$.

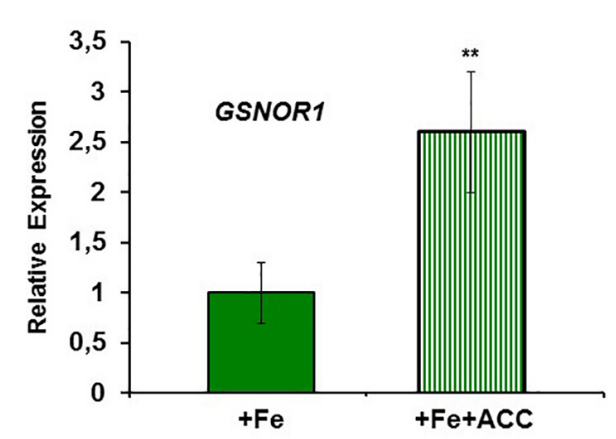

FIGURE 9 | Effect of ACC on GSNOR1 expression in roots of Arabidopsis WT Columbia plants. Plants were grown in nutrient solution with $40 \mu \mathrm{M} F e(+\mathrm{Fe})$ and half of them treated with $1 \mu \mathrm{M} \mathrm{ACC}$, final concentration (+Fe+ACC), $24 \mathrm{~h}$ before harvest. Relative expression was calculated in relation to $+\mathrm{Fe}$. Data represent the mean \pm SE of three independent biological replicates. Significant differences from the + Fe treatment are indicated: ${ }^{* *} P<0.05$.

ethylene synthesis (and signaling) in roots does not depend on total root Fe content (these mutants accumulate high levels of $\mathrm{Fe}$ inside roots under Fe sufficiency; García et al., 2013 and references therein) but on LODIS levels, that depend on the OPT3 transporter.

Since heavy metals can enhance ethylene production and the expression of ethylene signaling genes (Keunen et al., 2016), it can be argued that the higher ACC content and the higher expression of ethylene-related genes in Fe-sufficient opt3-2 roots (and in Fe-sufficient frd3-3 and nas $4 x-1$ roots) could be caused by the high accumulation of metals described in some of these mutants. Against this argument, we have to say that, in our experiments, plants were grown with low levels of metals and they did not present leaf toxicity symptoms (Figure 3B). Moreover, after the work of Vert et al. (2002) it is known that the accumulation of metals in mutants that present constitutive activation of IRT1 expression, such as opt3-2 (Stacey et al., 2008) and frd3 (Rogers and Guerinot, 2002), is mainly due to the broad substrate range 

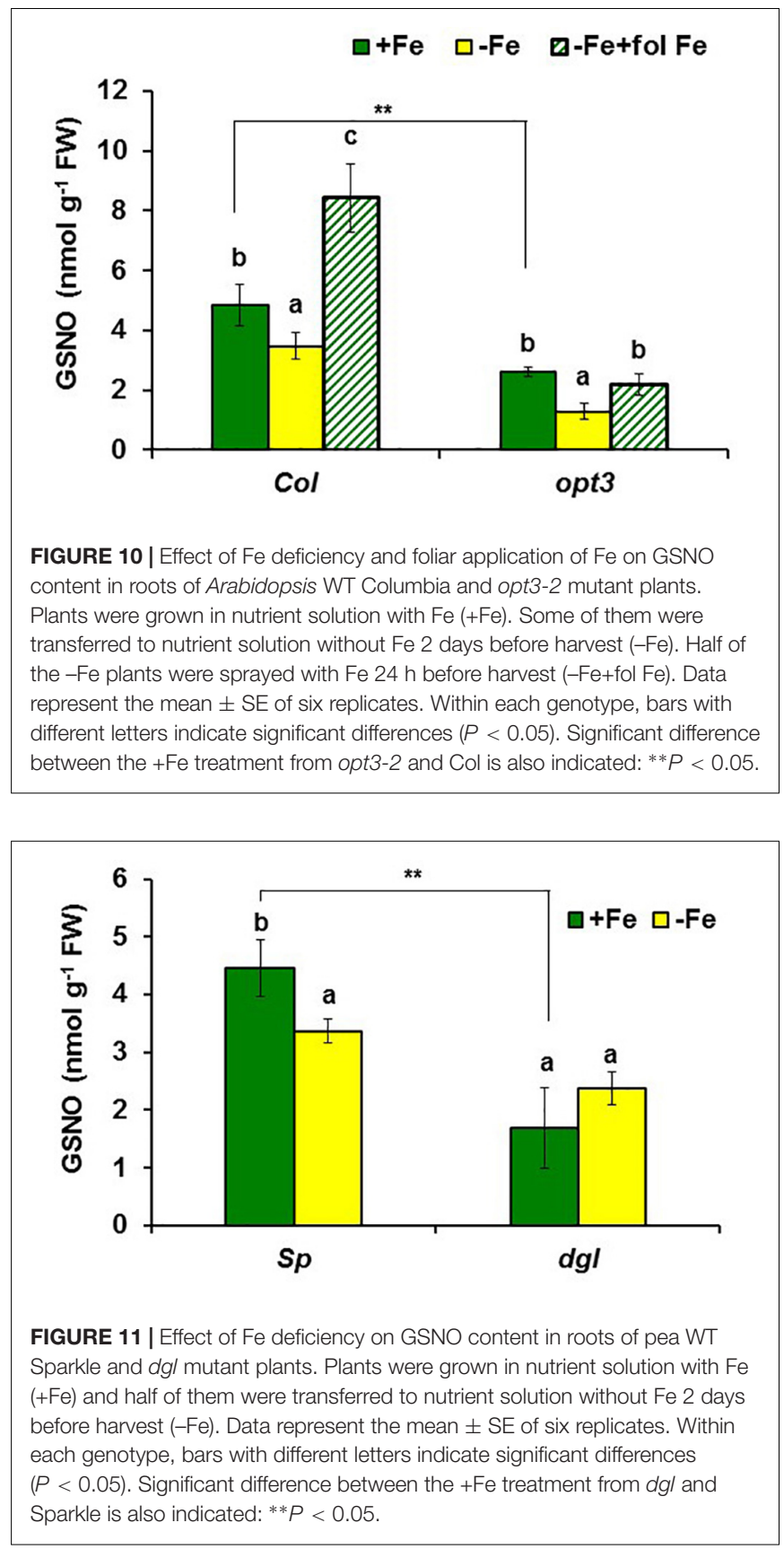

of this transporter. IRT1 can transport several divalent metals, besides $\mathrm{Fe}^{2+}$, such as $\mathrm{Mn}^{2+}, \mathrm{Zn}^{2+}$, or $\mathrm{Cd}^{2+}$ (Korshunova et al.,
1999; Lucena et al., 2006). Since IRT1 expression is activated by ethylene (García et al., 2010; Lingam et al., 2011; Blum et al., 2014; Marín-de la Rosa et al., 2014), the most probable sequence is: (1) enhanced ethylene production $\rightarrow(2)$ activation of IRT1 expression $\rightarrow$ (3) accumulation of metals, and not: (1) accumulation of metals $\rightarrow$ (2) enhanced ethylene production $\rightarrow$ (3) activation of IRT1 expression. No accumulation of metals would occur without IRT1 activity. In supporting this view, it should be noted that the inhibition of ethylene synthesis with cobalt, a potent ethylene inhibitor, drastically inhibited IRT1 expression and the accumulation of metals in tomato plants (Lucena et al., 2006) and in pea brz mutant plants (also named E107, has constitutive activation of Fe responses; Romera et al., 1996, 2015). Taken together, all these results suggest that ethylene is necessary for IRT1 up-regulation and, consequently, for the acquisition and accumulation of metals. This does not discard that, if plants accumulate high levels of metals, they could cause a further increase of ethylene production, but as a later side effect.

Similarly to ethylene, GSH (Table 2) and NO also increase in Fe-deficient WT roots, where they have been implicated in the activation of Fe deficiency responses (Zaharieva and Abadía, 2003; Zaharieva et al., 2004; Graziano and Lamattina, 2007; Bacaicoa et al., 2009; Chen et al., 2010; García et al., 2011; Koen et al., 2012; Shanmugam et al., 2015; Kailasam et al., 2018). However, results in this work show that GSNO content in WT roots, in contrast to its precursors GSH and NO, decreases upon Fe deficiency (Figures 10, 11), which agrees with previous results (Shanmugam et al., 2015; Kailasam et al., 2018). Since GSNOR reduces GSNO to GSSG and other byproducts, like hydroxylamine and $\mathrm{H}_{3} \mathrm{~N}$ (Leterrier et al., 2011; Corpas et al., 2013; Kubienová et al., 2014), we wanted to know whether GSNOR increases in Fe deficient roots. In fact, GSNOR1 expression and activity in roots increased early after Fe deficiency and slightly before the up-regulation of Fe acquisition genes (Figures 5, 6). This suggests that GSNOR could be responsible for the lower GSNO contents found in Fe-deficient WT roots (Figures 10, 11; Shanmugam et al., 2015; Kailasam et al., 2018). GSNOR1 is highly responsive to the $\mathrm{Fe}$ status of the plant but, as for ethylene (see above), the results indicate that its up-regulation does not depend on the total root $\mathrm{Fe}$ content but on LODIS levels. First, the foliar application of $\mathrm{Fe}$ greatly increased GSNO content in Fe-deficient Columbia roots (probably by decreasing GSNOR activity, as seen in Figure 6) but hardly in $\mathrm{Fe}$ deficient roots of the LODIS-deficient mutant opt3-2 (Figure 10), which is impaired in the movement of Fe from shoots to

TABLE 2 | Effect of Fe deficiency on GSH (glutathione) concentration in roots and leaves of Arabidopsis WT Columbia and opt3-2 mutant plants.

\begin{tabular}{|c|c|c|c|c|c|}
\hline Compound & Genotype & \multicolumn{2}{|c|}{ Roots } & \multicolumn{2}{|c|}{ Leaves } \\
\hline $\mathrm{GSH}\left(\mathrm{nmol} \cdot \mathrm{g}^{-1} \mathrm{FW}\right)$ & Col & $53 \pm 4$ & $144 \pm 1^{* *}$ & $146 \pm 10$ & $137 \pm 8$ \\
\hline
\end{tabular}

Plants were grown in nutrient solution with $\mathrm{Fe}(+\mathrm{Fe})$ and half of them transferred to nutrient solution without $\mathrm{Fe}(-\mathrm{Fe})$ during the last 2 days. Data represent the mean $\pm \mathrm{SE}$ of six replicates. Significant differences from the $+F$ e treatment are indicated: ${ }^{*} P<0.01$. 


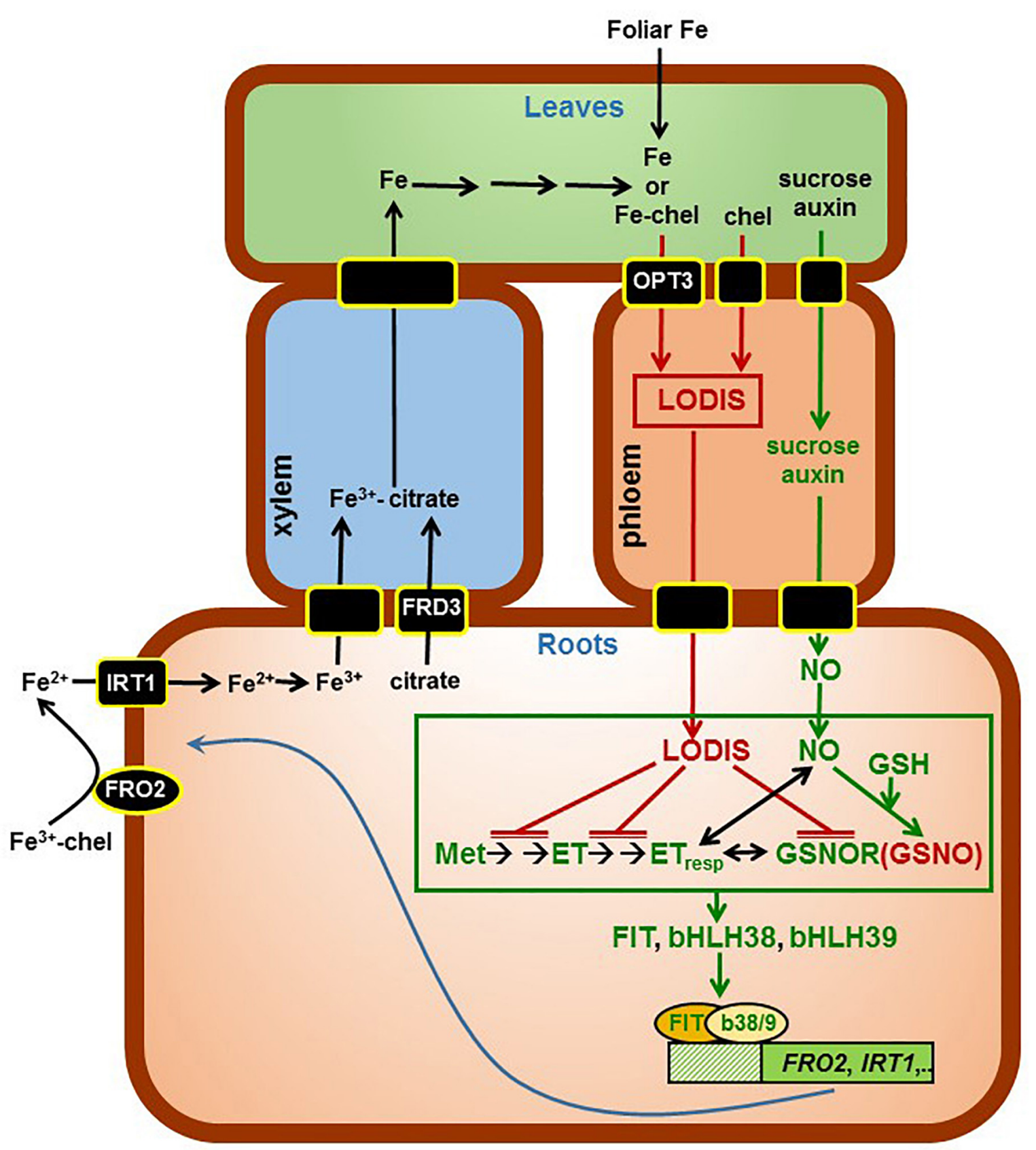

FIGURE 12 | Working Model to explain the role of LODIS on the regulation of Fe acquisition genes. Once inside roots, Fe is translocated to leaves through the xylem, bound to citrate (provided by the FRD3 transporter). In shoots, some Fe (either as free ions or in chelated form) can enter the phloem through the OPT3 transporter, and moves back to roots bound to a chelating agent (forming LODIS). In roots, LODIS could negatively affect ethylene synthesis and signaling, and GSNOR expression and/or activity, which can lead to enhanced GSNO. Besides LODIS, which would act as a repressor of Fe responses, some other shoot signals, like sucrose and auxin, would act as activators of Fe responses through NO (Lin et al., 2016). The possible relationship of ethylene, NO, GSH, GSNOR, and GSNO is depicted in more detail in Figure 13. In green are components whose expression, activity and/or content is known to increase under Fe deficiency while in red are components whose expression, activity, and/or content is known to increase under Fe sufficiency. chel, chelating agent; GSH, glutathione; GSNO, S-nitrosogluthatione; GSNOR, GSNOR reductase; ET, ethylene; ETresp, ethylene response; Met, methionine ( $\rightarrow$ : promotion; $\bar{T}$ : inhibition).

roots (García et al., 2013; Mendoza-Cózatl et al., 2014; Zhai et al., 2014). Second, Fe-sufficient opt3-2 roots presented much higher GSNOR1 expression (and less GSNO content) than Fesufficient Columbia roots (Figures 7, 10). Similarly, a lower
GSNO content was found in Fe-sufficient roots of the pea LODIS-deficient mutant $d g l$ than in Fe-sufficient roots of its WT Sparkle (Figure 11). All these results clearly suggest that GSNOR expression (and consequently GSNO content) 
does not depend on total root $\mathrm{Fe}$ content but on LODIS levels.

The results described above clearly show that the higher NO accumulation found in Fe-deficient WT roots (Graziano and Lamattina, 2007; Chen et al., 2010; García et al., 2011) is associated with lower GSNO contents (Figures 10, 11). Similar results have been found by others in roots of Arabidopsis plants subjected to Fe deficiency (Shanmugam et al., 2015; Kailasam et al., 2018), to arsenic stress (Leterrier et al., 2012) or to Cd stress (Liu et al., 2018). In the two latter cases, the lower GSNO levels have been associated with a higher GSNOR activity (Leterrier et al., 2012; Liu et al., 2018), which also occurs under other abiotic stress conditions (Corpas et al., 2008; Airaki et al., 2012; Kubienová et al., 2014; Cheng et al., 2015). In relation to $\mathrm{Cd}$ stress, it is important to mention that $\mathrm{Cd}$ can cause $\mathrm{Fe}$ deficiency (Alcántara et al., 1994 and references therein) and induce Fe deficiency responses (Yoshihara et al., 2006). In our work, besides Fe deficiency, GSNOR1 expression also increased under $\mathrm{P}$ or S deficiency (Figure 8), which indicates that GSNOR could be implicated in the responses to different nutritional deficiencies. The higher NO accumulation associated with a lower GSNO content in Fe-deficient roots suggests that NO and GSNO do not have the same exact roles, probably because they have different lifetime and location. NO lifetime is relatively short (less than $10 \mathrm{~s}$ ) while GSNO is a relatively stable store for NO and might constitute a vehicle for its long-distance transport (Corpas et al., 2011; Malik et al., 2011), mediating a signaling function throughout a process of $S$-transnitrosation between GSNO and proteins (Corpas et al., 2013). In relation to their location, both NO and GSNO can present different cellular and intracellular location (Malik et al., 2011; Shi et al., 2015). NO production is associated with phloem cells (Corpas et al., 2004; Gaupels et al., 2008) and, since the GSNOR protein is also located in the phloem (Rustérucci et al., 2007), it is possible that GSNO could be a vehicle to transmit the NOsignal out of the phloem. In agreement with the distinct role of NO and GSNO, Yun et al. (2016) have found that both of them act differently in the responses associated with plant immunity. In relation to Fe deficiency responses in dicot plants, Kailasam et al. (2018) have recently shown, by using chemical screening, that GSNO probably affects the regulation of $\mathrm{Fe}$ acquisition genes through FIT while NO could affect them through other TFs, such as bHLH38 and bHLH39. It should be noted that the SKB1 protein, implicated in bHLH38 and bHLH39 expression (Fan et al., 2014), can be nitrosylated (Hu et al., 2015). In this way, it is possible that GSNO, through the nitrosylation/de-nitrosylation of SKB1, could affect the expression of these Ib bHLH TFs (Fan et al., 2014; Lucena et al., 2015). In supporting this view, bHLH38, bHLH39, and bHLH1OO are up-regulated in leaves of the Arabidopsis gsnor1-3 mutant, which has altered GSNO content (Xu et al., 2013).

Results in this work, along with previous results (García et al., 2013), suggest that LODIS can inhibit both ethylene synthesis and signaling, and GSNOR expression and activity in roots (see Working Model in Figure 12). The inhibition of GSNOR would increase GSNO content in roots, which agrees with the higher GSNO contents found in Fe-sufficient WT roots or in roots of Fe-deficient WT plants treated with foliar Fe (Figures 10, 11; Shanmugam et al., 2015; Kailasam et al., 2018). After all these results, several questions arise: what is the nature of LODIS?; is there any relationship between GSNO(NO), GSH and ethylene?; is there any relationship between $\mathrm{GSNO}(\mathrm{NO}) /$ ethylene and the putative Fe sensor BTS? In relation to the nature of LODIS, it is unknown at present. Although Zhai et al. (2014) found that OPT3 can transport $\mathrm{Fe}^{2+}$ ions when expressed in Xenopus oocytes, the substrate of OPT3 remains controversial because the Fe transport function of OPT3 could not be confirmed in other experiments (Mendoza-Cózatl et al., 2014). Even if Fe enters the phloem through OPT3 as free ions, it would be difficult for Fe to move in this way, and probably the Fe ions would be chelated (Figure 12; see section "Introduction"). OPT3 does not mediate GSH transport in S. cerevisiae (Zhai et al., 2014), as other OPT transporters did (Zhang et al., 2016 and references therein). However, the higher leaf GSH content found in the opt3-2 mutant in relation to Columbia, under Fe sufficiency (Table 2) or under Cd treatment (Akmakjian, 2011), supports the idea of LODIS being a compound related, directly or indirectly, to GSH, such as GS-Fe-NO or NICs (Ramírez et al., 2011; Rahmanto et al., 2012; Darbani et al., 2013; Buet and Simontacchi, 2015). Nonetheless, this idea deserves further research.

In relation to the possible interactions between GSNO(NO), GSH and ethylene, it should be said that, besides the mutual and positive influence between $\mathrm{NO}$ and ethylene already described in previous works (Figure 13; García et al., 2011; Romera et al., 2011; Liu et al., 2017), GSNO(NO), GSH and ethylene could also interact at other levels. GSH can enhance ethylene production by affecting some ethylene synthesis enzymes (Datta et al., 2015). On the other hand, the relationship between GSNO and ethylene can be feasible because both GSNOR (Rustérucci et al., 2007; see above) and several ethylene synthesis enzymes, like 5-methilthioribose kinase (induced in Fe-deficient roots; García et al., 2010; Romera et al., 2017 and references therein), are located in the phloem (Pommerrenig et al., 2011). Moreover, enzymes implicated in ethylene synthesis, such as SAM synthetases (also named methionine adenosyltransferases; Sauter et al., 2013), can be inhibited by $S$-nitrosylation (Lindermayr et al., 2006; Freschi, 2013). Since GSNO has been implicated in the reversible $S$-nitrosylation of proteins (Malik et al., 2011; Begara-Morales et al., 2015; Zaffagnini et al., 2016), it is possible that higher GSNO levels (such as those found in Fe-sufficient WT roots: Figures 10, 11) could contribute to $S$-nitrosylation of ethylene synthesis enzymes and, consequently, to the inhibition of ethylene synthesis (Figure 13). By contrast, lower GSNO levels (such as those found in Fe-deficient WT roots or in Fe-sufficient opt3-2 and $d g l$ roots: Figures 10, 11) could contribute to de-nitrosylation of ethylene synthesis enzymes and, consequently, to promotion of ethylene synthesis (Figures 1B, 2). In supporting this view, silencing GSNOR in Nicotiana attenuata, which leads to higher GSNO content, decreased the herbivore-induced accumulation of ethylene (Wünsche et al., 2011). Reciprocally to the possible 


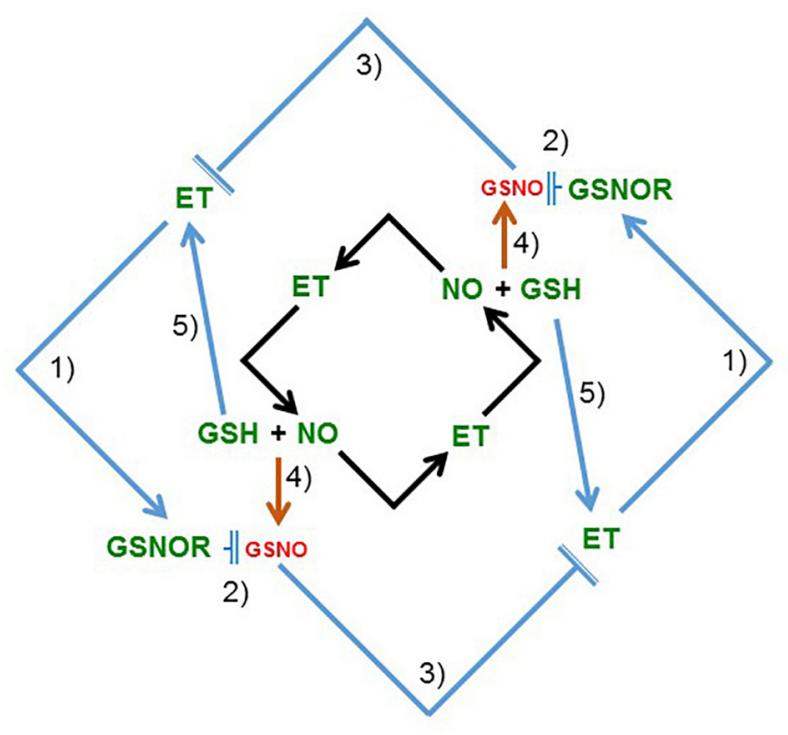

FIGURE 13 | Model to explain the relationship between ethylene and NO, and between ethylene and GSNOR (GSNO), in roots. In previous works, and also in this work (see Figure 2 and Table 2), it has been shown that ethylene, NO, and GSH increase in Fe-deficient roots of dicot plants (see section "Introduction"). Moreover, it has been shown that ethylene can influence NO accumulation in roots and vice versa (black arrows; García et al., 2011; Liu et al., 2017). In this work, it has been found that ethylene and GSNOR increase under low LODIS accumulation in roots (see Figure 12). After that, a new model, complementary of the previous one, is proposed (blue arrows) suggesting mutual possible interactions among ethylene, GSNOR, GSH and NO: (1) ethylene can upregulate GSNOR (see Figure 9); (2) GSNOR can decrease GSNO (Corpas et al., 2013); (3) Low GSNO can unblock ethylene synthesis since nitrosylation of SAM synthetase by GSNO can inhibit ethylene synthesis (Lindermayr et al., 2006; Freschi, 2013; see section "Discussion"); (4) GSNO derives from NO and GSH (Corpas et al., 2013); (5) GSH can enhance ethylene production (Datta et al., 2015). In green are components whose expression, activity and/or content is known to increase under Fe deficiency while in red are components whose content is known to decrease under Fe deficiency. GSH, glutathione; GSNO, S-nitrosoglutathione; GSNOR, GSNO reductase; ET, ethylene; NO, nitric oxide ( $\rightarrow$ : promotion; $\overline{\mathrm{T}}$ : inhibition).

influence of GSNO(NO) on ethylene synthesis, ethylene (ACC) could decrease GSNO content by activating GSNOR1 expression (Figures 9, 13). GSNOR1 expression is also influenced by other hormones, such as salicylic acid which activates it or jasmonic acid which down-regulates it (Díaz et al., 2003). Very recently, it has been found that ethylene (ACC) can increase NO content by activating enzymes involved in its synthesis, such as nitrate reductase and nitric oxide synthaselike (Liu et al., 2017). This would imply that ethylene could simultaneously increase NO accumulation and decrease GSNO content (Figure 13).

Finally, in the relationship between GSNO(NO)/ethylene and the putative Fe sensor BTS, some results suggest that the BTS protein could act upstream of ethylene. The SAM1 gene, encoding a SAM synthetase (involved in ethylene synthesis; Sauter et al., 2013), is up-regulated in the bts-3 mutant (Hindt et al., 2017). In the same way, several genes whose expression is activated by ethylene, such as FIT, bHLH38, bHLH39, MYB72, BGLU42, FRO2, IRT1, FRD3, and NAS2 (García et al., 2010), are up-regulated in bts-3 roots under $\mathrm{Fe}$ sufficient conditions (Hindt et al., 2017). In rice, roots from HRZ-knockdown plants have higher jasmonate content than the WT ones when grown under Fe sufficiency (Kobayashi et al., 2016). All these results suggest that BTS/HRZs may act as sensors of LODIS, as suggested by Kobayashi and Nishizawa (2014), and depending on its binding state could then affect the synthesis of hormones, like ethylene or jasmonate.

\section{CONCLUSION}

The results presented in this work, along with already published results, suggest that shoots, through LODIS, play an important role in the regulation of Fe acquisition genes in roots, which is depicted in the Working Model of Figure 12. According to this model, under Fe sufficiency, sufficient Fe goes to leaves and enters the phloem to form LODIS, which travels to roots and represses ethylene synthesis and signaling, and GSNOR expression and activity, which leads to increased GSNO content. GSNO(NO), $\mathrm{GSH}$, and ethylene can interact in different ways (see above paragraph and Figure 13). Moreover, a high GSNO content can compromise auxin signaling and transport (Shi et al., 2015), which is important because auxin has also been involved in the regulation of Fe responses, where is intimately interrelated with ethylene and NO (Chen et al., 2010; Romera et al., 2011, 2017). Under Fe deficiency, the lack of LODIS derepresses ethylene synthesis and signaling, and GSNOR expression and activity, which leads to decreased GSNO content. All this would cause the up-regulation of Fe acquisition genes.

This working model could open the way to better understand the role of shoots and roots in the regulation of Fe deficiency responses. According to this model, LODIS would have a double role in the regulation of Fe acquisition genes, by controlling both ethylene synthesis and signaling (Figure 12). This double role of LODIS would explain why the ethylene overproducer mutant eto and the treatments with ethylene did not activate the expression of Fe acquisition genes when plants are grown with high levels of Fe (Lucena et al., 2015): despite high ethylene levels, LODIS would block ethylene action. All the results discussed in this work imply that the relationship of GSH, NO and GSNO with ethylene, with GSNOR, with LODIS and with the expression of Fe acquisition genes is complex and deserves further research.

\section{AUTHOR'S NOTE}

A very recent work by Grillet et al. (2018) shows that some peptides (IRON MAN or IMA peptides) associated with the phloem, and preferentially expressed in leaves, could be implicated in the regulation of $\mathrm{Fe}$ responses in roots. The upregulation of $\mathrm{Fe}$ responses in lines overexpressing genes encoding these peptides and their ability to bind $\mathrm{Fe}^{2+}$ suggest that these peptides could act either as activators (unbound IMA peptides) or repressors (IMA peptides bound to $\mathrm{Fe}$ ) of the 
responses. Perhaps LODIS is related to the ratio between IMA peptides bound to Fe and unbound IMA peptides.

\section{AUTHOR CONTRIBUTIONS}

FR and MG designed the experiments after discussions with PB, RP-V, and EA. MG and CL conducted the laboratory work. FC and MG determined GSNOR, GSNO, and GSH. ÁZ, EB, and JG-M determined ACC. FR, MG, RP-V, and EA wrote the manuscript that was improved by the other authors.

\section{FUNDING}

This work was supported by the European Regional Development Fund from the European Union, the 'Ministerio de Economía

\section{REFERENCES}

Airaki, M., Leterrier, M., Mateos, R. M., Valderrama, R., Chaki, M., Barroso, J. B., et al. (2012). Metabolism of reactive oxygen species and reactive nitrogen species in pepper (Capsicum annuum L.) plants under low temperature stress. Plant Cell Environ. 35, 281-295. doi: 10.1111/j.1365-3040.2011.02310.x

Airaki, M., Sánchez-Moreno, L., Leterrier, M., Barroso, J. B., Palma, J. M., and Corpas, F. J. (2011). Detection and quantification of S-nitrosoglutathione (GSNO) in pepper (Capsicum annuum L.) plant organs by LC-ES/MS. Plant Cell Physiol. 52, 2006-2015. doi: 10.1093/pcp/pcr133

Akmakjian, G. Z. (2011). Long-Distance Cadmium Transport and Regulation of Heavy Metal Stress Responses in Arabidopsis thaliana. Master Science thesis, University of California, San Diego, CA.

Alcántara, E., Romera, F. J., De La Guardia, M. D., and Cañete, M. (1994). Effects of heavy metals on both induction and function of root $\mathrm{Fe}$ (III) reductase in Fe-deficient cucumber (Cucumis sativus L.) plants. J. Exp. Bot. 281, 1893-1898. doi: 10.1093/jxb/45.12.1893

Bacaicoa, E., Mora, V., Zamarreño, A. M., Fuentes, M., Casanova, E., and GarcíaMina, J. M. (2011). Auxin: a major player in the shoot-to-root regulation of root Fe-stress physiological responses to Fe deficiency in cucumber plants. Plant Physiol. Biochem. 49, 545-556. doi: 10.1016/j.plaphy.2011.02.018

Bacaicoa, E., Zamarreño, A. M., Leménager, D., Baigorri, R., and García-Mina, J. M. (2009). Relationship between the hormonal balance and the regulation of iron deficiency stress responses in cucumber. J. Am. Soc. Hortic. Sci. 134, 589-601.

Barberon, M., Vermeer, J. E. M., De Bellis, D., Wang, P., Naseer, S., Andersen, T. G., et al. (2016). Adaptation of root function by nutrient-induced plasticity of endodermal differentiation. Cell 164, 1-13. doi: 10.1016/j.cell.2015.12.021

Bauer, P., Ling, H. Q., and Guerinot, M. L. (2007). Fit, the FER-like iron deficiency induced transcription factor in Arabidopsis. Plant Physiol. Biochem. 45, 260-261. doi: 10.1016/j.plaphy.2007.03.006

Begara-Morales, J. C., Sánchez-Calvo, B., Chaki, M., Mata-Pérez, C., Valderrama, R., Padilla, M. N., et al. (2015). Differential molecular response of monodehydro- ascorbate reductase and glutathione reductase by nitration and S-nitrosylation. J. Exp. Bot. 66, 5983-5996. doi: 10.1093/jxb/ erv306

Blum, A., Brumbarova, T., Bauer, P., and Ivanov, R. (2014). Hormone influence on the spatial regulation of IRT1 expression in iron-deficient Arabidopsis thaliana roots. Plant Signal. Behav. 9:e28787. doi: 10.4161/psb.28787

Brumbarova, T., Bauer, P., and Ivanov, R. (2015). Molecular mechanisms governing Arabidopsis iron uptake. Trends Plant Sci. 20, 124-133. doi: 10.1016/j.tplants. 2014.11.004

Buet, A., and Simontacchi, M. (2015). Nitric oxide and plant iron homeostasis. Ann. N. Y. Acad. Sci. 1340, 39-46. doi: 10.1111/nyas.12644

Chen, W. W., Yang, J. L., Qin, C., Jin, C. W., Mo, J. H., Ye, T., et al. (2010). Nitric oxide acts downstream of auxin to trigger root ferric-chelate reductase activity in response to iron deficiency in Arabidopsis thaliana. Plant Physiol. 154, 810-819. doi: 10.1104/pp.110.161109 y Competitividad' (Projects AGL2013-40822-R and AGL201565104-P) and the 'Junta de Andalucía' (Research Groups AGR115, BIO159, and BIO192).

\section{ACKNOWLEDGMENTS}

We thank Dr. Brian M. Waters, of the University of Nebraska (Lincoln, NE, United States), for the English correction and valuable suggestions in the editing of the manuscript. We thank Dr. Michael Grusak for providing seeds of the Sparkle [dgl,dgl] mutant, the Arabidopsis Biological Resource Center for providing seeds of the frd3-3 mutant and Dr. Stacey for providing seeds of the opt3-2 mutant. We also thank Inmaculada Montilla and Carmelo Ruíz-Torres for their excellent technical support.

Cheng, T., Chen, J., Ef, A., Wang, P., Wang, G., Hu, X., et al. (2015). Quantitative proteomics analysis reveals that S-nitrosoglutathione reductase (GSNOR) and nitric oxide signaling enhance poplar defense against chilling stress. Planta 242, 1361-1390. doi: 10.1007/s00425-015-2374-5

Corpas, F. J., Alché, J. D., and Barroso, J. B. (2013). Current overview of S-nitrosoglutathione (GSNO) in higher plants. Front. Plant Sci. 4:126. doi: 10.3389/ fpls.2013.00126 doi: 10.3389/fpls.2013.00126

Corpas, F. J., Barroso, J. B., Carreras, A., Quirós, M., León, A. M., RomeroPuertas, M. C., et al. (2004). Cellular and subcellular localization of endogenous nitric oxide in young and senescent pea plants. Plant Physiol. 136, 2722-2733. doi: 10.1104/pp.104.042812

Corpas, F. J., Chaki, M., Fernández-Ocaña, A., Valderrama, R., Palma, J. M., Carreras, A., et al. (2008). Metabolism of reactive nitrogen species in pea plants under abiotic stress conditions. Plant Cell Physiol. 49, 1711-1722. doi: 10.1093/ pcp/pcn 144

Corpas, F. J., Leterrier, M., Valderrama, R., Airaki, M., Chaki, M., Palma, J. M., et al. (2011). Nitric oxide imbalance provokes a nitrosative response in plants under abiotic stress. Plant Sci. 181, 604-611. doi: 10.1016/j.plantsci.2011.04.005

Darbani, B., Briat, J. F., Holm, P. B., Husted, S., Noeparvar, S., and Borg, S. (2013). Dissecting plant iron homeostasis under short and long-term iron fluctuations. Biotechnol. Adv. 31, 1292-1307. doi: 10.1016/j.biotechadv.2013.05.003

Datta, R., Kumar, D., Sultana, A., Hazra, S., Bhattacharyya, S., and Chattopadhyay, S. (2015). Glutathione regulates 1-Aminocyclopropane-1Carboxylate synthase transcription via WRKY33 and 1-Aminocyclopropane-1Carboxylate Oxidase by modulating messenger RNA stability to induce ethylene synthesis during stress. Plant Physiol. 169, 2963-2981. doi: 10.1104/pp.15.01543

Díaz, M., Achkor, H., Titarenko, E., and Martínez, M. C. (2003). The gene encoding glutathione-dependent formaldehyde dehydrogenase/GSNO reductase is responsive to wounding, jasmonic acid and salicylic acid. FEBS Lett. 543, 136-139. doi: 10.1016/S0014-5793(03)00426-5

Fan, H., Zhang, Z., Wang, N., Cui, Y., Sun, H., Liu, Y., et al. (2014). SKB1/PRMT5mediated histone H4R3 dimethylation of Ib subgroup bHLH genes negatively regulates iron homeostasis in Arabidopsis thaliana. Plant J. 77, 209-221. doi: $10.1111 /$ tpj. 12380

Freschi, L. (2013). Nitric oxide and phytohormone interactions: current status and perspectives. Front. Plant Sci. 4:398. doi: 10.3389/fpls.2013.00398

García, M. J., Lucena, C., Romera, F. J., Alcántara, E., and Pérez-Vicente, R. (2010). Ethylene and nitric oxide involvement in the up-regulation of key genes related to iron acquisition and homeostasis in Arabidopsis. J. Exp. Bot. 61, 3885-3899. doi: 10.1093/jxb/erl189

García, M. J., Romera, F. J., Lucena, C., Alcántara, E., and Pérez-Vicente, R. (2015). Ethylene and the regulation of physiological and morphological responses to nutrient deficiencies. Plant Physiol. 169, 51-60. doi: 10.1104/pp.15.00708

García, M. J., Romera, F. J., Stacey, M. G., Stacey, G., Villar, E., Alcántara, E., et al. (2013). Shoot to root communication is necessary to control the expression of iron-acquisition genes in strategy I plants. Planta 237, 65-75. doi: 10.1007/ s00425-012-1757-0 
García, M. J., Suárez, V., Romera, F. J., Alcántara, E., and Pérez-Vicente, R. (2011). A new model involving ethylene, nitric oxide and $\mathrm{Fe}$ to explain the regulation of Fe-acquisition genes in Strategy I plants. Plant Physiol. Biochem. 49, 537-544. doi: 10.1016/j.plaphy.2011.01.019

Gaupels, F., Furch, A. C. U., Will, T., Mur, L. A. J., Kogel, K. H., and van Bel, A. J. E. (2008). Nitric oxide generation in Vicia faba phloem cells reveals them to be sensitive detectors as well as possible systemic transducers of stress signals. New Phytol. 178, 634-646. doi: 10.1111/j.1469-8137.2008.02388.x

Gayomba, S. R., Zhai, Z., Jung, H., and Vatamaniuk, O. K. (2015). Local and systemic signaling of iron status and its interactions with homeostasis of other essential elements. Front. Plant Sci. 6:716. doi: 10.3389/fpls.2015.00716

Graziano, M., Beligni, M. V., and Lamattina, L. (2002). Nitric oxide improves internal iron availability in plants. Plant Physiol. 130, 1852-1859. doi: 10.1104/ pp.009076

Graziano, M., and Lamattina, L. (2007). Nitric oxide accumulation is required for molecular and physiological responses to iron deficiency in tomato roots. Plant J. 52, 949-960. doi: 10.1111/j.1365-313X.2007.03283.x

Grillet, L., Lan, P., Li, W., Mokkapati, G., and Schmidt, W. (2018). Iron man, a ubiquitous family of peptides that control iron transport in plants. bioRxiv [Preprint]. doi: 10.1101/351635

Gutiérrez-Carbonell, E., Lattanzio, G., Albacete, A., Ríos-Ruiz, J. J., Kehr, J., Abadía, A., et al. (2015). Effects of Fe deficiency on the protein profile of Brassica napus phloem sap. Proteomics 15, 3835-3853. doi: 10.1002/pmic.201400464

Han, B., Yang, Z., Samma, M. K., Wang, R., and Shen, W. (2013). Systematic validation of candidate reference genes for qRT-PCR normalization under iron deficiency in Arabidopsis. Biometals 26, 403-413. doi: 10.1007/s10534-0139623-5

Hindt, M. N., Akmakjian, G. Z., Pivarski, K. L., Punshon, T., Baxter, I., Salt, D. E., et al. (2017). Brutus and its paralogs, BTS LIKE1 and BTS LIKE2, encode important negative regulators of the iron deficiency response in Arabidopsis thaliana. Metallomics 9, 876-890. doi: 10.1039/c7mt00152e

Hu, J., Huang, X., Chen, L., Sun, X., Lu, C., Zhang, L., et al. (2015). Site-specific nitrosoproteomic identification of endogenously S-nitrosylated proteins in Arabidopsis. Plant Physiol. 167, 1731-1746. doi: 10.1104/pp.15.00026

Ivanov, R., Brumbarova, T., and Bauer, P. (2012). Fitting into the harsh reality: regulation of iron-deficiency responses in dicotyledonous plants. Mol. Plant 5, 27-42. doi: 10.1093/mp/ssr065

Kailasam, S., Wang, Y., Lo, J. C., Chang, H. F., and Yeh, K. C. (2018). S-nitrosoglutathione works downstream of nitric oxide to mediate iron deficiency signaling in Arabidopsis. Plant J. 94, 157-168. doi: 10.1111/tpj.13850

Keunen, E., Schellingen, K., Vangronsveld, J., and Cuypers, A. (2016). Ethylene and metal stress: small molecule, big impact. Front. Plant Sci. 7:23. doi: 10.3389/fpls. 2016.00023

Khan, M. A., Castro-Guerrero, N. A., McInturf, S. A., Nguyen, N. T., Dame, A. N., Wang, J., et al. (2018). Changes in iron availability in Arabidopsis are rapidly sensed in the leaf vasculature and impaired sensing leads to opposite transcriptional programs in leaves and roots. Plant Cell Environ. doi: 10.1111/ pce.13192 [Epub ahead of print].

Klatte, M., Schuler, M., Wirtz, M., Fink-Straube, C., Hell, R., and Bauer, P. (2009). The analysis of Arabidopsis nicotianamine synthase mutants reveals functions for nicotianamine in seed iron loading and iron deficiency responses. Plant Physiol. 150, 257-271. doi: 10.1104/pp.109.136374

Kobayashi, T., Itai, R. N., Senoura, T., Oikawa, T., Ishimaru, Y., Ueda, M., et al. (2016). Jasmonate signaling is activated in the very early stages of iron deficiency responses in rice roots. Plant Mol. Biol. 91, 533-547. doi: 10.1007/s11103-0160486-3

Kobayashi, T., and Nishizawa, N. K. (2012). Iron uptake, translocation, and regulation in higher plants. Annu. Rev. Plant Biol. 63, 131-152. doi: 10.1146/ annurev-arplant-042811-10552

Kobayashi, T., and Nishizawa, N. K. (2014). Iron sensors and signals in response to iron deficiency. Plant Sci. 224, 36-43. doi: 10.1016/j.plantsci.2014.04.002

Koen, E., Szymańska, K., Klinguer, A., Dobrowolska, G., Besson-Bard, A., and Wendehenne, D. (2012). Nitric oxide and glutathione impact the expression of iron uptake- and iron transport-related genes as well as the content of metals in A. thaliana plants grown under iron deficiency. Plant Signal. Behav. 7, 1246-1250. doi: 10.4161/psb.21548

Korshunova, Y. O., Eide, D., Clark, W. G., Guerinot, M. L., and Pakarasi, H. B. (1999). The IRT1 protein from Arabidopsis thaliana is a metal transporter with a broad substrate range. Plant Mol. Biol. 40, 37-44. doi: 10.1023/A: 1026438615520

Krüger, C., Berkowitz, O., Stephan, U. W., and Hell, R. (2002). A metal-binding member of the late embryogenesis abundant family transports iron in the phloem of Ricinus communis L. J. Biol. Chem. 277, 25062-25069. doi: 10.1074/ jbc.M201896200

Kubienová, L., Tichá, T., Jahnová, J., Luhová, L., Mieslerová, B., and Petrivalský, M. (2014). Effect of abiotic stress stimuli on S-nitrosoglutathione reductase in plants. Planta 239, 139-146. doi: 10.1007/s00425-013-1970-5

Kumar, R. K., Chu, H. H., Abundis, C., Vasques, K., Rodriguez, D. C., Chia, J. C., et al. (2017). Iron-nicotianamine transporters are required for proper long distance iron signaling. Plant Physiol. 175, 1254-1268. doi: 10.1104/pp.17.00821

Leaden, L., Pagani, M. A., Balparda, M., Busi, M. V., and Gomez-Casati, D. F. (2016). Altered levels of AtHSCB disrupts iron translocation from roots to shoots. Plant Mol. Biol. 92, 613-628. doi: 10.1007/s11103-016-0537-9

Leterrier, M., Airaki, M., Palma, J. M., Chaki, M., Barroso, J. B., and Corpas, F. J. (2012). Arsenic triggers the nitric oxide (NO) and S-nitrosoglutathione (GSNO) metabolism in Arabidopsis. Environ. Pollut. 166, 136-143. doi: 10.1016/j.envpol. 2012.03.012

Leterrier, M., Chaki, M., Airaki, M., Valderrama, R., Palma, J. M., Barroso, J. B., et al. (2011). Function of S-nitrosoglutathione reductase (GSNOR) in plant development and under biotic/abiotic stress. Plant Signal. Behav. 6, 789-793. doi: $10.4161 /$ psb.6.6.15161

Li, W., and Lan, P. (2017). The understanding of the plant iron deficiency responses in strategy I plants and the role of ethylene in this process by omic approaches. Front. Plant Sci. 8:40. doi: 10.3389/fpls.2017.00040

Li, X., Zhang, H., Ai, Q., Liang, G., and Yu, D. (2016). Two bHLH transcription factors, bHLH34 and bHLH104, regulate iron homeostasis in Arabidopsis thaliana. Plant Physiol. 170, 2478-2493. doi: 10.1104/pp.15.01827

Liang, G., Zhang, H., Li, X., Ai, Q., and Yu, D. (2017). bHLH transcription factor bHLH115 regulates iron homeostasis in Arabidopsis thaliana. J. Exp. Bot. 68, 1743-1755. doi: 10.1093/jxb/erx043

Lin, X. Y., Ye, Y. Q., Fan, S. K., Jin, C. W., and Zheng, S. J. (2016). Increased sucrose accumulation regulates iron-deficiency responses by promoting auxin signaling in Arabidopsis plants. Plant Physiol. 170, 907-920. doi: 10.1104/pp.15.01598

Lin, Y., Yang, L., Paul, M., Zu, Y., and Tang, Z. (2013). Ethylene promotes germination of Arabidopsis seed under salinity by decreasing reactive oxygen species: evidence for the involvement of nitric oxide simulated by sodium nitroprusside. Plant Physiol. Biochem. 73, 211-218. doi: 10.1016/j.plaphy.2013. 10.003

Lindermayr, C., Saalbach, G., Bahnweg, G., and Durner, J. (2006). Differential inhibition of Arabidopsis methionine adenosyltransferases by protein S-nitrosylation. J. Biol. Chem. 281, 4285-4291. doi: 10.1074/jbc.M511635200

Lingam, S., Mohrbacher, J., Brumbarova, T., Potuschak, T., Fink-Straube, C., Blondet, E., et al. (2011). Interaction between the bHLH transcription factor FIT and the Ethilene Insensitive3/ Ethylene Insensitive3-like1 reveals molecular linkage between the regulation of iron acquisition and ethylene signaling in Arabidopsis. Plant Cell 23, 1815-1829. doi: 10.1105/tpc.111.084715

Liu, M., Liu, X. X., He, X. L., Liu, L. J., Wu, H., Tang, C. X., et al. (2017). Ethylene and nitric oxide interact to regulate the magnesium deficiency-induced root hair development in Arabidopsis. New Phytol. 213, 1242-1256. doi: 10.1111/ nph.14259

Liu, S., Yang, R., Tripathi, D. K., Li, X., He, W., Wu, M., et al. (2018). The interplay between reactive oxygen and nitrogen species contributes in the regulatory mechanism of the nitro-oxidative stress induced by cadmium in Arabidopsis. J. Hazard. Mater. 344, 1007-1024. doi: 10.1016/j.jhazmat.2017.12.004

Lubkowitz, M. (2011). The oligopeptide transporters: a small gene family with a diverse group of substrates and functions? Mol. Plant 4, 407-415. doi: 10.1093/ $\mathrm{mp} / \mathrm{ssr} 004$

Lucena, C., Romera, F. J., García, M. J., Alcántara, E., and Pérez-Vicente, R. (2015). Ethylene participates in the regulation of Fe deficiency responses in Strategy I plants and in rice. Front. Plant Sci 6:1056. doi: 10.3389/fpls.2015.01056

Lucena, C., Romera, F. J., Rojas, C. L., García, M. J., Alcántara, E., and PérezVicente, R. (2007). Bicarbonate blocks the expression of several genes involved in the physiological responses to Fe deficiency of Strategy I plants. Funct. Plant Biol. 34, 1002-1009. doi: 10.1071/FP07136

Lucena, C., Waters, B. M., Romera, F. J., García, M. J., Morales, M., Alcántara, E., et al. (2006). Ethylene could influence ferric reductase, iron transporter and 
$\mathrm{H}+$-ATPase gene expression by affecting FER (or FER-like) gene activity. J. Exp. Bot. 57, 4145-4154. doi: 10.1093/jxb/erl189

Malik, S. I., Hussain, A., Yun, B. W., Spoel, S. H., and Loake, G. J. (2011). GSNOR-mediated de-nitrosylation in the plant defence response. Plant Sci. 181, 540-544. doi: 10.1016/j.plantsci.2011.04.004

Marentes, E., and Grusak, M. A. (1998). Mass determination of low-molecularweight proteins in phloem sap using matrix-assisted laser desorption/ionisation time-of-flight mass spectrometry. J. Exp. Bot. 49, 903-911.

Marín-de la Rosa, N., Sotillo, B., Miskolczi, P., Gibbs, D. J., Vicente, J., Carbonero, P., et al. (2014). Large-scale identification of gibberellin-related transcription factors defines group VII Ethylene Response Factors as functional DELLA partners. Plant Physiol. 166, 1022-1032. doi: 10.1104/pp.114.244723

Maurer, F., Müller, S., and Bauer, P. (2011). Suppression of Fe deficiency gene expression by jasmonate. Plant Physiol. Biochem. 49, 530-536. doi: 10.1016/j. plaphy.2011.01.025

Meiser, J., Lingam, S., and Bauer, P. (2011). Post-transcriptional regulation of the Fe deficiency bHLH transcription factor FIT is affected by iron and nitric oxide. Plant Physiol. 157, 2154-2166. doi: 10.1104/pp.111.183285

Mendoza-Cózatl, D. G., Xie, Q., Akmakjian, G. Z., Jobe, T. O., Patel, A., Stacey, M. G., et al. (2014). OPT3 is a component of the iron-signaling network between leaves and roots and misregulation of opt3 leads to an over-accumulation of cadmium in seeds. Mol. Plant 7, 1455-1469. doi: 10.1093/mp/ssu067

Mora, V., Baigorri, R., Bacaicoa, E., Zamarreño, A. M., and García-Mina, J. M. (2012). The humic acid-induced changes in the root concentration of nitric oxide, IAA and ethylene do not explain the changes in root architecture caused by humic acid in cucumber. Environ. Exp. Bot. 76, 24-32. doi: 10.1016/j. envexpbot.2011.10.001

Pfaffl, M. W. (2001). A new mathematical model for relative quantification in real-time RT-PCR. Nucleic Acids Res. 29:e45. doi: 10.1093/nar/29.9.e45

Pommerrenig, B., Feussner, K., Zierer, W., Rabinovych, V., Klebl, F., Feussner, I., et al. (2011). Phloem-specific expression of Yang cycle genes and identification of novel Yang cycle enzymes in Plantago and Arabidopsis. Plant Cell 23, 1904-1919. doi: 10.1105/tpc.110.079657

Rahmanto, Y. S., Kalinowski, D. S., Lane, D. J. R., Lok, H. C., Richardson, V., and Richardson, D. R. (2012). Nitrogen monoxide (NO) storage and transport by dinitrosyl-dithiol-iron complexes: long-lived NO that is trafficked by interacting proteins. J. Biol. Chem. 287, 6960-6968. doi: 10.1074/jbc.R111. 329847

Ramírez, L., Bartoli, C. G., and Lamattina, L. (2013). Glutathione and ascorbic acid protect Arabidopsis plants against detrimental effects of iron deficiency. J. Exp. Bot. 64, 3169-3178. doi: 10.1093/jxb/ert153

Ramírez, L., Simontacchi, M., Murgia, I., Zabaleta, E., and Lamattina, L. (2011). Nitric oxide, nitrosyl iron complexes, ferritin and frataxin: a well equipped team to preserve plant iron homeostasis. Plant Sci. 181, 582-592. doi: 10.1016/ j.plantsci.2011.04.006

Rogers, E. E., and Guerinot, M. L. (2002). FRD3, a member of the multidrug and toxin efflux family, controls iron deficiency responses in Arabidopsis. Plant Cell 14, 1787-1799. doi: 10.1105/tpc.001495

Romera, F. J., García, M. J., Alcántara, E., and Pérez-Vicente, R. (2011). Latest findings about the interplay or auxin, ethylene and nitric oxide in the regulation of Fe deficiency responses by Strategy I plants. Plant Signal. Behav. 6, 167-170. doi: $10.4161 /$ psb.6.1.14111

Romera, F. J., Lucena, C., García, M. J., Alcántara, E., and Pérez-Vicente, R. (2015). "Regulation of Fe deficiency responses in wt pea and some of its mutants (brz and dgl)," in Pisum sativum: Cultivation, Functional Properties and Health Benefits, ed. S. Becket (New York, NY: Nova Science Publishers Inc.), 1-20.

Romera, F. J., Lucena, C., García, M. J., Alcántara, E., and Pérez-Vicente, R. (2017). "The role of ethylene and other signals in the regulation of Fe deficiency responses by dicot plants," in Stress Signaling in Plants: Genomics and Proteomics Perspectives, Vol. 2, ed. M. Sarwat (Dordrecht: Springer), 277-300.

Romera, F. J., Welch, R. M., Norvell, W. A., and Schaefer, S. C. (1996). Iron requirement for and effects of promoters and inhibitors of ethylene action on stimulation of Fe(llI)-chelate reductase in roots of Strategy I species. Biometals 9, 45-50. doi: 10.1007/BF00188089

Römheld, V., and Marschner, H. (1986). Mobilization of iron in the rhizosphere of different plant species. Adv. Plant Nutr. 2, 155-204.

Roschzttardtz, H., Séguéla-Arnaud, M., Briat, J. F., Vert, G., and Curie, C. (2011). The FRD3 citrate effluxer promotes iron nutrition between simplistically disconnected tissues throughout Arabidopsis development. Plant Cell 23, 2725-2737. doi: 10.1105/tpc.111.088088

Rustérucci, C., Espunya, M. C., Díaz, M., Chabannes, M., and Martínez, M. C. (2007). S-nitrosoglutathione reductase affords protection against pathogens in Arabidopsis, both locally and systemically. Plant Physiol. 143, 1282-1292. doi: $10.1104 /$ pp.106.091686

Sakamoto, A., Ueda, M., and Morikawa, H. (2002). Arabidopsis glutathionedependent formaldehyde dehydrogenase is an S-nitrosoglutathione reductase. FEBS Lett. 515, 20-24. doi: 10.1016/S0014-5793(02)02414-6

Sauter, M., Moffatt, B., Saechao, M. C., Hell, R., and Wirtz, M. (2013). Methionine salvage and S-adenosylmethionine: essential links between sulfur, ethylene and polyamine biosynthesis. Biochem. J. 451, 145-154. doi: 10.1042/BJ20121744

Schuler, M., Rellán-Álvarez, R., Fink-Straube, C., Abadía, J., and Bauer, P. (2012). Nicotianamine functions in the phloem-based transport of iron to sink organs, in pollen development and pollen tube growth in Arabidopsis. Plant Cell 24, 2380-2400. doi: 10.1105/tpc.112.099077

Séguéla, M., Briat, J. F., Vert, G., and Curie, C. (2008). Cytokinins negatively regulate the root iron uptake machinery in Arabidopsis through a growthdependent pathway. Plant J. 55, 289-300. doi: 10.1111/j.1365-313X.2008. 03502.x

Shanmugam, V., Wang, Y. W., Tsednee, M., Karunakaran, K., and Yeh, K. C. (2015). Glutathione plays an essential role in nitric oxide-mediated irondeficiency signaling and iron-deficiency tolerance in Arabidopsis. Plant J. 84, 464-477. doi: 10.1111/tpj.13011

Shen, C., Yang, Y., Liu, K., Zhang, L., Guo, H., Sun, T., et al. (2016). Involvement of endogenous salicylic acid in iron-deficiency responses in Arabidopsis. J. Exp. Bot. 67, 4179-4193. doi: 10.1093/jxb/erw196

Shi, Y. F., Wang, D. L., Wang, C., Culler, A. H., Kreiser, M. A., Suresh, J., et al. (2015). Loss of GSNOR1 function leads to compromised auxin signaling and polar auxin transport. Mol. Plant 8, 1350-1365. doi: 10.1016/j.molp.2015.04.008

Stacey, M. G., Patel, A., McClain, W. E., Mathieu, M., Remley, M., Rogers, E. E., et al. (2008). The Arabidopsis AtOPT3 protein functions in metal homeostasis and movement of iron to developing seeds. Plant Physiol. 146, 589-601. doi: $10.1104 /$ pp.107.108183

Vert, G., Grotz, N., Dedaldechamp, F., Gaymard, F., Guerinot, M. L., Briat, J. F., et al. (2002). IRT1, an Arabidopsis transporter essential for iron uptake from the soil and for plant growth. Plant Cell 14, 1223-1233. doi: 10.1105/tpc.001388

Wang, N., Cui, Y., Liu, Y., Fan, H., Du, J., Huang, Z., et al. (2013). Requirement and functional redundancy of Ib subgroup bHLH proteins for iron deficiency responses and uptake in Arabidopsis thaliana. Mol. Plant 6, 503-513. doi: $10.1093 / \mathrm{mp} / \mathrm{sss} 089$

Waters, B. M., Lucena, C., Romera, F. J., Jester, G. G., Wynn, A. N., Rojas, C. L., et al. (2007). Ethylene involvement in the regulation of the $\mathrm{H}^{+}$-ATPase CsHA1 gene and of the new isolated ferric reductase CsFRO1 and iron transporter CsIRT1 genes in cucumber plants. Plant Physiol. Biochem. 45, 293-301. doi: 10.1016/j.plaphy.2007.03.011

Wintz, H., Fox, T., Wu, Y. Y., Feng, V., Chen, W., Chang, H. S., et al. (2003). Expression profiles of Arabidopsis thaliana in mineral deficiencies reveal novel transporters involved in metal homeostasis. J. Biol. Chem. 278, 47644-47653. doi: 10.1074/jbc.M309338200

Wünsche, H., Baldwin, I. T., and Wu, J. (2011). S-nitrosoglutathione reductase (GSNOR) mediates the biosynthesis of jasmonic acid and ethylene induced by feeding of the insect herbivore Manduca sexta and is important for jasmonate-elicited responses in Nicotiana attenuata. J. Exp. Bot. 62, 4605-4616. doi: $10.1093 /$ jxb/err171

Xu, S., Guerra, D., Lee, U., and Vierling, E. (2013). S-nitrosoglutathione reductases are low-copy number, cysteine-rich proteins in plants that control multiple developmental and defense responses in Arabidopsis. Front. Plant Sci. 4:430. doi: 10.3389/fpls.2013.00430

Yang, Y., Ou, B., Zhang, J., Si, W., Gu, H., Qin, G., et al. (2014). The Arabidopsis mediator subunit MED16 regulates iron homeostasis by associating with EIN3/EIL1 through subunit MED25. Plant J. 77, 838-851. doi: 10.1111/tpj. 12440

Ye, L., Li, L., Wang, L., Wang, S., Li, S., Du, J., et al. (2015). MPK3/MPK6 are involved in iron deficiency-induced ethylene production in Arabidopsis. Front. Plant Sci. 6:953. doi: 10.3389/fpls.2015.00953

Yoshihara, T., Hodoshima, H., Miyano, Y., Shoji, K., Shimada, H., and Goto, F. (2006). Cadmium inducible Fe deficiency responses observed from macro and 
molecular views in tobacco plants. Plant Cell Rep. 25, 365-373. doi: 10.1007/ s00299-005-0092-3

Yuan, Y. X., Wu, H. L., Wang, N., Li, J., Zhao, W. N., Du, J., et al. (2008). FIT interacts with AtbHLH038 and AtbHLH039 in regulating iron uptake gene expression for iron homeostasis in Arabidopsis. Cell Res. 18, 385-397. doi: $10.1038 / \mathrm{cr} .2008 .26$

Yun, B. W., Skelly, M. J., Yin, M., Yu, M., Mun, B. G., Lee, S. U., et al. (2016). Nitric oxide and S-nitrosoglutathione function additively durin plant immunity. New Phytol. 211, 516-526. doi: 10.1111/nph.13903

Zaffagnini, M., DeMia, M., Morisse, S., Di Giacinto, N., Marchand, C. H., Maes, A., et al. (2016). Protein S-nitrosylation in photosynthetic organisms: a comprehensive overview with future perspectives. Biochim. Biophys. Acta 1864, 952-966. doi: 10.1016/j.bbapap.2016.02.006

Zaharieva, T. B., and Abadía, J. (2003). Iron deficiency enhances the levels of ascorbate, glutathione and related enzymes in sugar beet roots. Protoplasma 221, 269-275. doi: 10.1007/s00709-002-0051-6

Zaharieva, T. B., Gogorcena, Y., and Abadía, J. (2004). Dynamics of metabolic responses to iron deficiency in sugar beet roots. Plant Sci. 166, 1045-1050. doi: 10.1016/j.plantsci.2003.12.017

Zhai, Z., Gayomba, S. R., Jung, H., Vimalakumari, N. K., Piñeros, M., Craft, E., et al. (2014). OPT3 is a phloem-specific iron transporter that is essential for systemic iron signaling and redistribution of iron and cadmium in Arabidopsis. Plant Cell 26, 2249-2264. doi: 10.1105/tpc.114.123737

Zhang, J., Liu, B., Li, M., Feng, D., Jin, H., Wang, P., et al. (2015). The bHLH transcription factor bHLH104 interacts with IAA-Leucine Resistant3 and modulates iron homeostasis in Arabidopsis. Plant Cell 27, 787-805. doi: 10.1105/tpc.114.132704

Zhang, Z., Xie, Q., Jobe, T. O., Kau, A. R., Wang, C., Li, Y., et al. (2016). Identification of AtOPT4 as a plant glutathione transporter. Mol. Plant 9, 481-484. doi: 10.1016/j.molp.2015.07.013

Conflict of Interest Statement: The authors declare that the research was conducted in the absence of any commercial or financial relationships that could be construed as a potential conflict of interest.

Copyright (C) 2018 García, Corpas, Lucena, Alcántara, Pérez-Vicente, Zamarreño, Bacaicoa, García-Mina, Bauer and Romera. This is an open-access article distributed under the terms of the Creative Commons Attribution License (CC BY). The use, distribution or reproduction in other forums is permitted, provided the original author(s) and the copyright owner(s) are credited and that the original publication in this journal is cited, in accordance with accepted academic practice. No use, distribution or reproduction is permitted which does not comply with these terms. 